\title{
barrage du Verney
}

\author{
Analyse de la fondation \\ Prévision du comportement à la mise en eau \\ Comparaison avec les mesures
}

\author{
Verney dam
}

\author{
Foundation analysis \\ Prediction of impounding performance \\ Comparison with field measurements \\ J. BERTRAND, ingénieur spécialiste, E. FROSSARD, ingénieur principal \\ Coyne et Bellier
}

\section{Résumé}

\begin{abstract}
Le barrage de Verney est un remblai d'alluvions de $42 \mathrm{~m}$ de hauteur, construit sur une fondation alluvio-morainique perméable, épaisse de $80 \mathrm{~m}$. Il est étanché par un masque amont en béton bitumineux, prolongé en fondation par une paroi moulée en béton plastique profonde de $46 \mathrm{~m}$.

Mise en place dès le début des travaux, une instrumentation dense a conduit à réviser et préciser l'image des caractéristiques et de la structure de la fondation, durant la construction. Cette fondation est apparue plus déformable et hétérogène que prévu.

Un modèle mathématique complet des ouvrages a été alors réalisé pour prévoir et contrôler le comportement à la mise en eau. Lors du remplissage, une concordance remarquable a été trouvée entre les mesures en place et les prévisions du calcul.
\end{abstract}

\section{Abstract}

Verney dam is a $42 \mathrm{~m}$ high embankment dam, built on a $80 \mathrm{~m}$ thick pervious glaciofluvial deposit. The dam is sealed by an upstream asphaltic facing, linked to a plastic-concrete diaphragm wall, in the foundation, down to $46 \mathrm{~m}$ depth.

Placed at the beginning of works, a dense instrumentation net led to some revision of foundation structure and characteristics, during dam construction. This foundation appeared more heterogeneous and softer than expected.

A complete mathematical model of the structure was then implemented, to predict and control dam behaviour at impounding. After reservoir filling, a remarkable accordance has been found between field measurements and model prediction. 


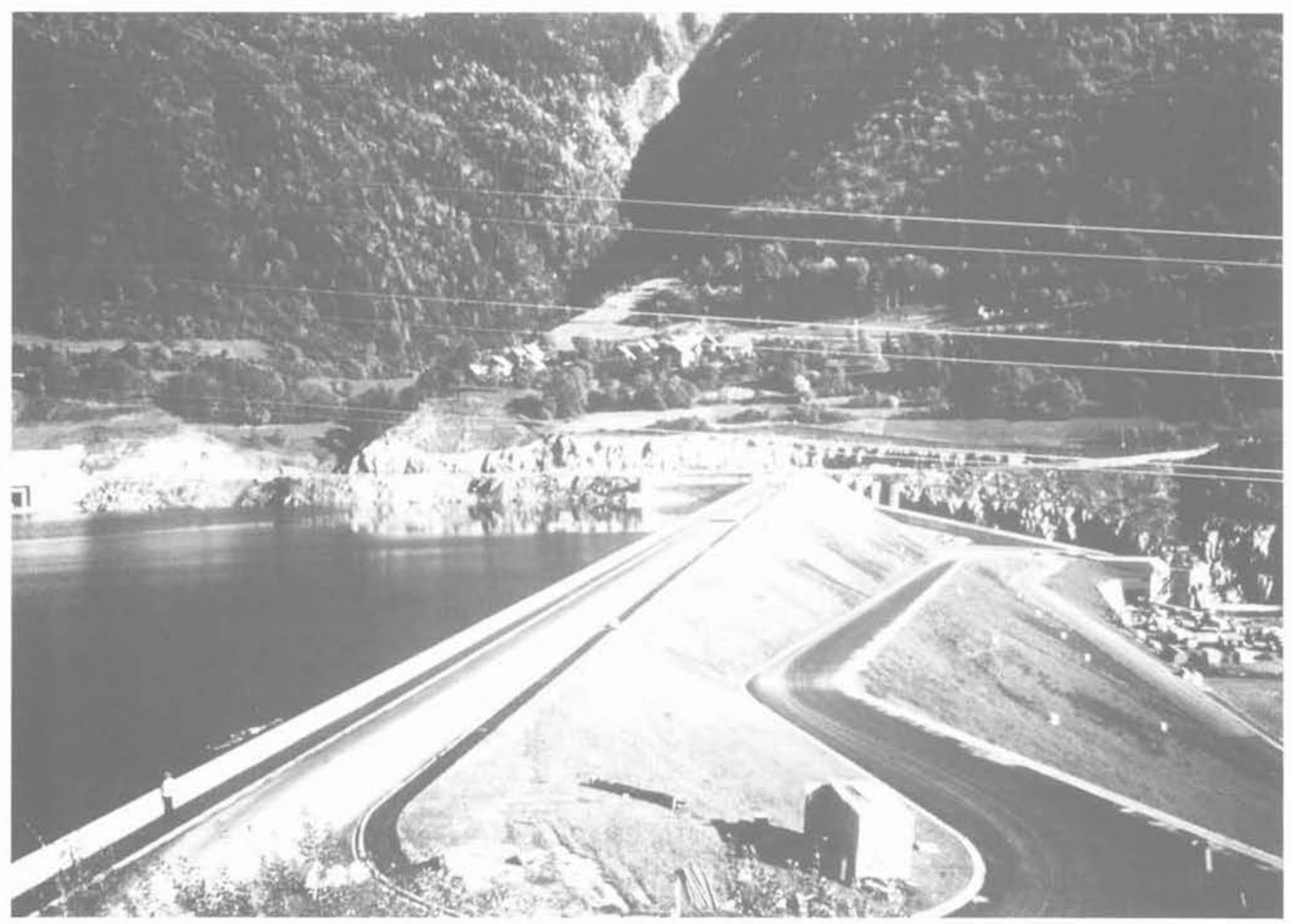

Fig. 1. - Le barrage de Verney en eau.

\section{PRÉSENTATION}

Située dans la vallée de l'Eau d'Olle, entre les massifs cristallins de Belledonne et des Grandes Rousses, la retenue du Verney (fig. 1) constitue le bassin inférieur de la station de transfert d'énergie par pompage de Grand Maison, réalisée par Électricité de France. (COSTE, POST, TARDIEU - 1982).

La retenue s'étend sur la plaine du Verney, une vallée creusée par les glaciers quaternaires dans les schistes cristallins du Lias, et dont le fond est rempli de dépôts fluvioglaciaires de forte épaisseur, atteignant $80 \mathrm{~m}$ à l'emplacement du barrage.

Les caractères essentiels du projet étaient :

- en premier lieu, une fondation profonde et perméable.

Les reconnaissances dans cette masse peu différenciée avaient conduit, non sans peine, à une première image de sa structure et de ses caractéristiques (fig. 2. Coupe transversale) :
- en partie supérieure, des alluvions constituées de sables, graviers et galets mêlés de quelques lentilles d'argile ou de silt, dont la perméabilité comprise en moyenne entre $10^{-4}$ et $10^{-3} \mathrm{~m} / \mathrm{s}$, atteint localement $10^{-5}$ à $10^{-2}$;

- en partie inférieure, des moraines à blocs mêlés d'argile grise peu perméables.

A partir d'essais à la plaque dans des puits Benoto et d'essais de laboratoire, les caractéristiques mécaniques avaient été estimées en moyenne aux valeurs suivantes :

$$
\begin{array}{ll}
\varnothing^{\prime} \leq 35^{\circ} & \mathrm{E}=150 \mathrm{MPa} \\
\mathrm{C}^{\prime}=0 & \nu=0,3
\end{array}
$$

- en second lieu, une organisation des ouvrages définie à partir des données précédentes (fig. 3. - Coupe type)

Le barrage est un remblai d'alluvions de $42 \mathrm{~m}$ de hauteur à étanchéité amont, réalisée par un masque souple en béton bitumineux, prolongé en fondation par 


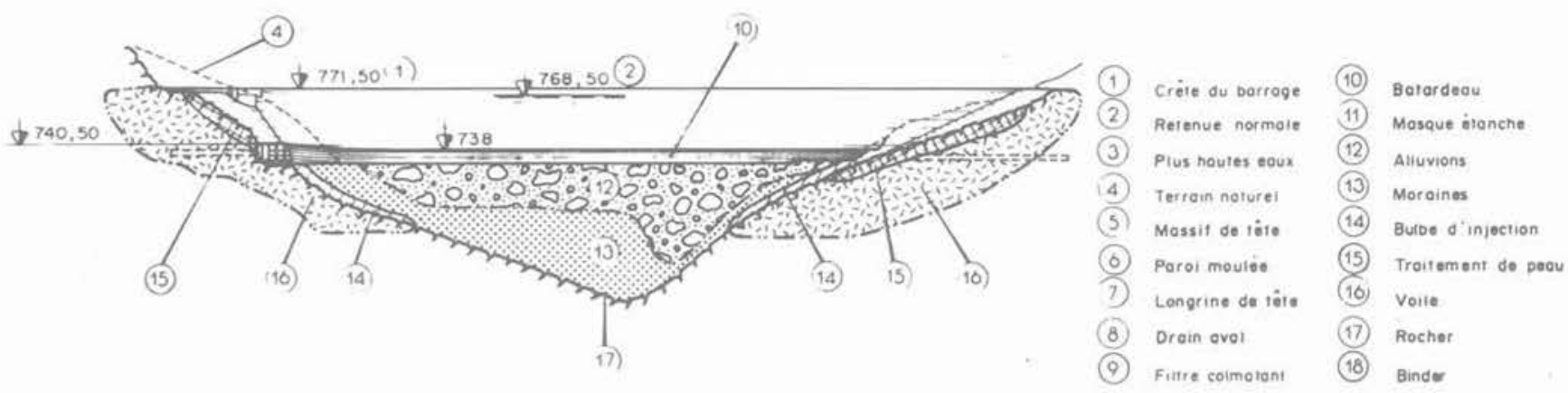

Fig. 2. - Coupe en travers de la vallée.

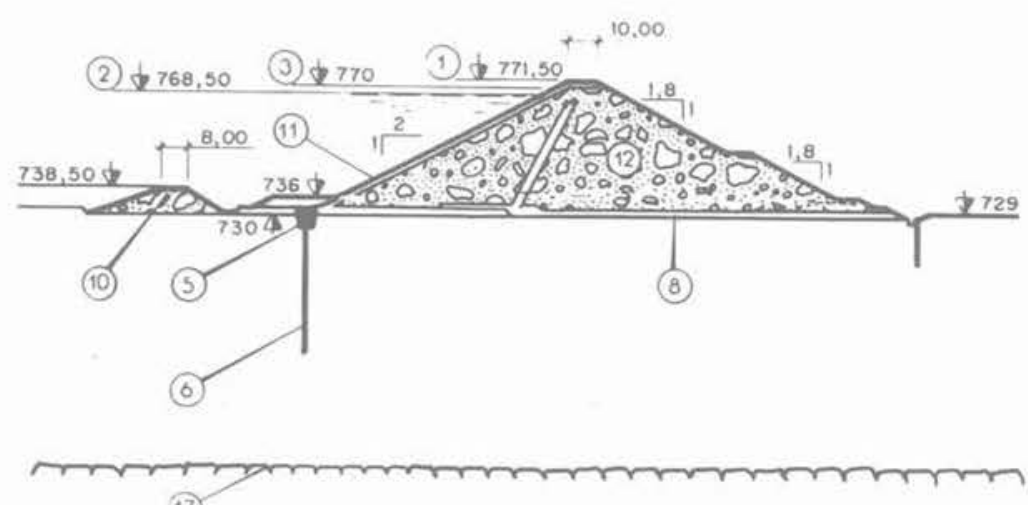

- Mosque detail Roccordement

(17)

Fig. 3. - Coupe type du barrage.

une paroi moulée en béton plastique de $46 \mathrm{~m}$ de profondeur (TARDIEU, 1981, 1984).

Sous la coupure étanche dans la fondation, un passage libre maintient l'alimentation de la nappe à l'aval.

Au pied amont du barrage, dans la zone de concentration des efforts de cisaillement exercés par l'ouvrage sur sa fondation, la partie supérieure de la paroi moulée constituait un point sensible, clairement identifié. Le masque étanche mince, fixé en son pied à la paroi moulée encastrée dans la fondation, et appuyé sur toute sa hauteur sur le remblai susceptible - à la mise en eau - de se déformer vers l'aval, constituait un second point sensible, quoique à moindre titre que le premier.

Le souci de s'assurer du bon fonctionnement des ouvrages, notamment par le suivi des déformations dans les zones sensibles, avait conduit à adjoindre au projet la mise en place d'une instrumentation dense (Groupe de travail du C.F.G.B., 1985).

Cette instrumentation comprend :

- un ensemble de dix piézomètres et douze cellules piézométriques en forage permettant le suivi de la nappe sous le barrage et à son voisinage, jusqu'à $60 \mathrm{~m}$ de profondeur dans la fondation (fig. 4 et 5);

- un clinomètre et deux extensomètres à 10 points de mesures installés dans des forages verticaux traversant la fondation jusqu'au bedrock, $80 \mathrm{~m}$ sous le barrage (fig. 5).

Le clinomètre et un extensomètre sont situés à 11,5 m à laval de la paroi moulée, le deuxième extensomètre étant dans laxe du barrage. Aucun appareil n'a été placé dans la paroi moulée pour éviter de perturber son comportement.

Cette instrumentation, mise en place dès le début des travaux, présentait en outre les avantages suivants, durant la construction même du barrage :

- apporter des précisions sur les caractéristiques hydrogéologiques et mécaniques de la fondation;

- guider l'adaptation éventuelle de certaines dispositions constructives ;

- permettre l'évaluation voire la prévision du comportement à la mise en eau. 

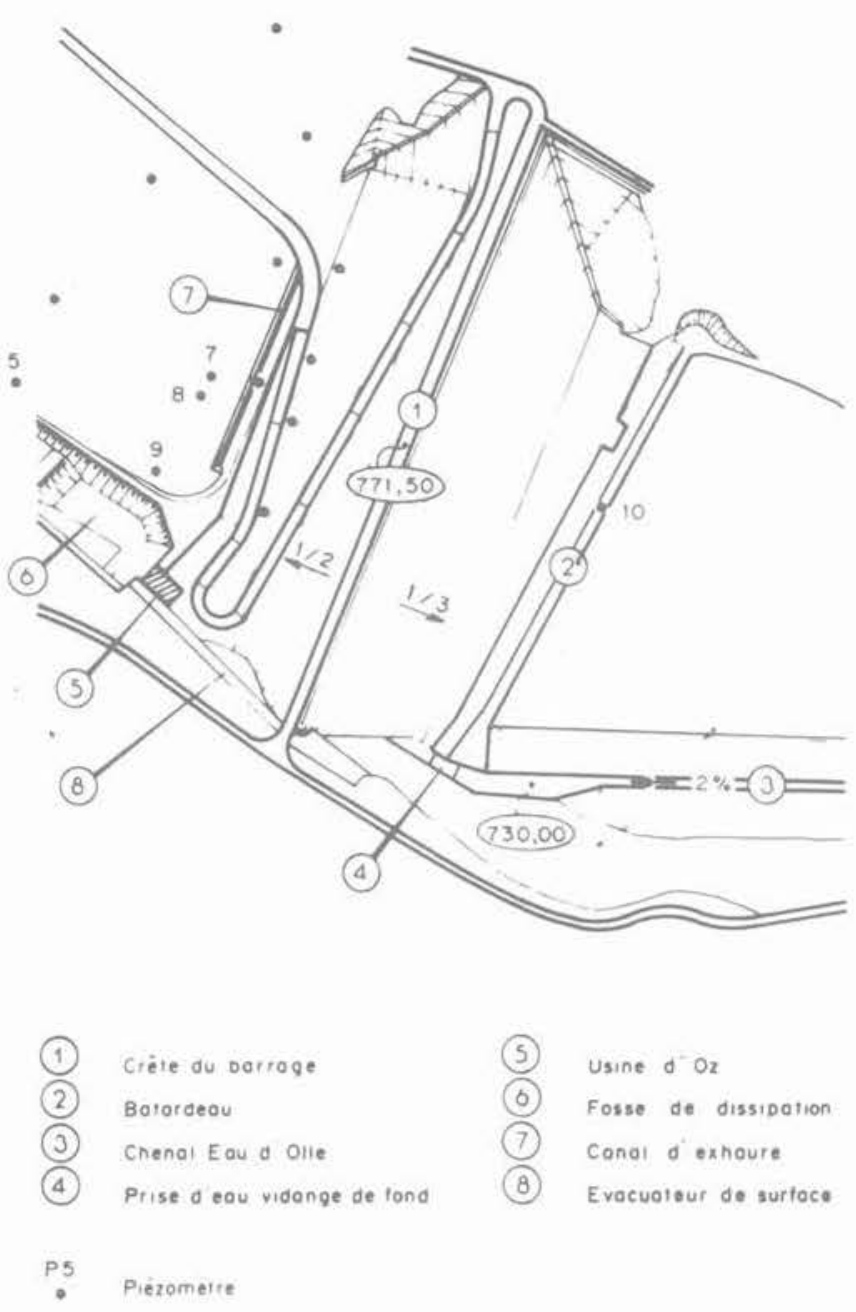

Fig. 4. - Implantation des piézomètres.
L'auscultation de la fondation a été complétée par le dispositif d'auscultation du barrage, installé au fur et à mesure de la construction, et qui consiste en un réseau de mires topographiques et d'un furet horizontal mis en place dans la partie inférieure du corps du barrage.

Durant la construction, l'analyse des informations nouvelles ainsi collectées a conduit à préciser et réviser l'image de la structure de la fondation et des caractéristiques hydrogéologiques et mécaniques. Pour l'essentiel, la fondation est apparue plus déformable et hétérogène que prévu.

Afin de s'assurer que cette déformabilité n'aurait pas de conséquence sur la tenue des organes d'étanchéité, il a été décidé de réaliser une étude complète pour prévoir le comportement à la mise en eau.

Un modèle mathématique, construit sur cette nouvelle image de la fondation, incorporant une loi de comportement mécanique non linéaire, a été alors calé sur les mesures réalisées durant la construction. Les prévisions faites au début de l'année 1984 à l'aide de ce modèle ont permis de conclure que le comportement des ouvrages resterait tout à fait acceptable lors de la mise en eau.

Les prédictions du calcul ont été confirmées par les mesures en place, avec une étonnante précision, à la mise en eau du barrage, achevée en janvier 1985.

L'exposé qui suit relate les éléments essentiels qui ont conduit à cette prévision :

- une première partie détaille l'élaboration de la nouvelle image de la fondation à partir des interprétations hydrogéologiques et mécaniques issues des mesures en place durant la construction ;

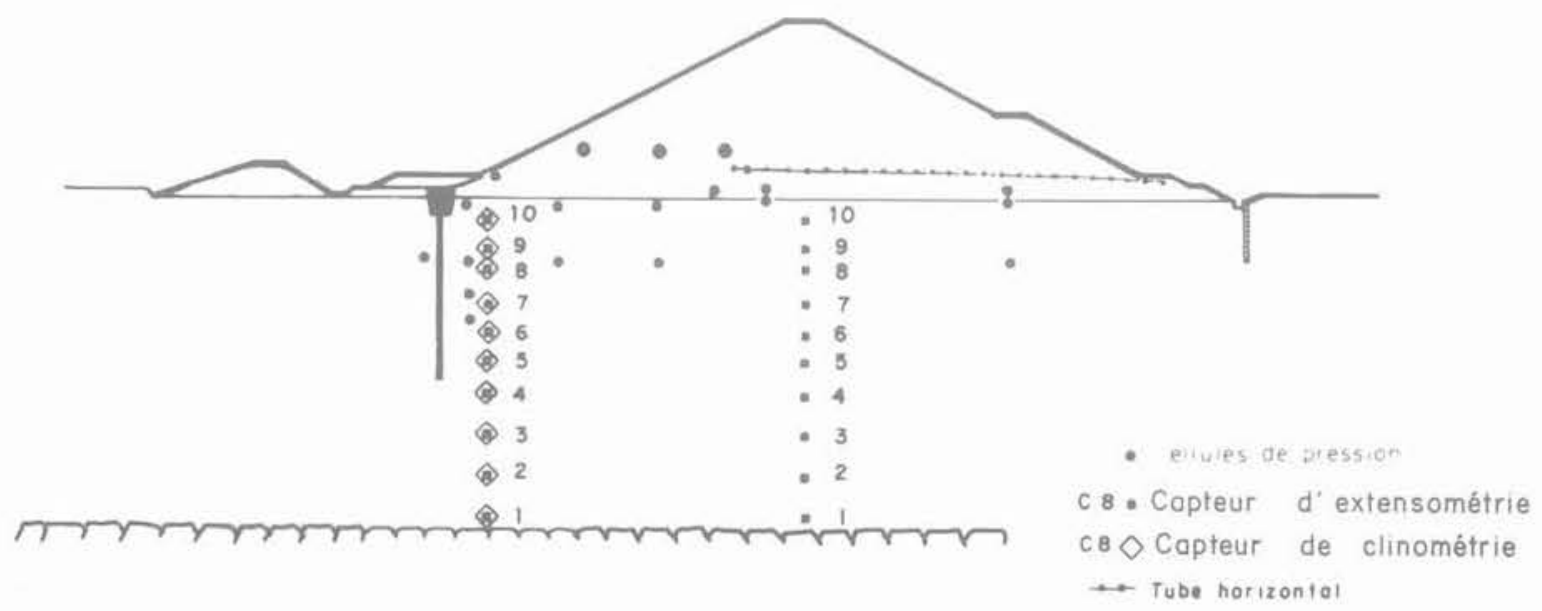

Fig. 5. - Dispositifs de mesure des pressions et des déformations. 
- une deuxième partie expose la modélisation mathématique réalisée. Cette partie donne un aperçu sur la loi de comportement non linéaire, mise au point pour la circonstance, et présente les étapes de calage des paramètres et de calcul du comportement jusqu'à la mise en eau, ainsi que leur comparaison avec les mesures.

\section{L'IMAGE DE LA FONDATION TIRÉE DES MESURES EN PLACE}

\subsection{L'analyse hydrogéologique}

L'objectif premier de cette analyse était de préciser les conditions de l'écoulement sous le barrage lors de sa mise en eau.
Afin d'exploiter au mieux les données disponibles, l'étude a été réalisée en trois étapes :

- élaboration d'une image hydrogéologique au moyen des données sur l'écoulement naturel avant travaux;

- contrôle de cette image au moyen des mesures réalisées durant les travaux. La fermeture de la paroi moulée donnant naissance à un écoulement transitoire fortement modifié par rapport à l'écoulement naturel stationnaire, la situation était particulièrement favorable à un tel contrôle:

- exploitation du modèle hydrogéologique, ainsi construit et contrôlé, en vue de la prévision du comportement à la mise en eau.

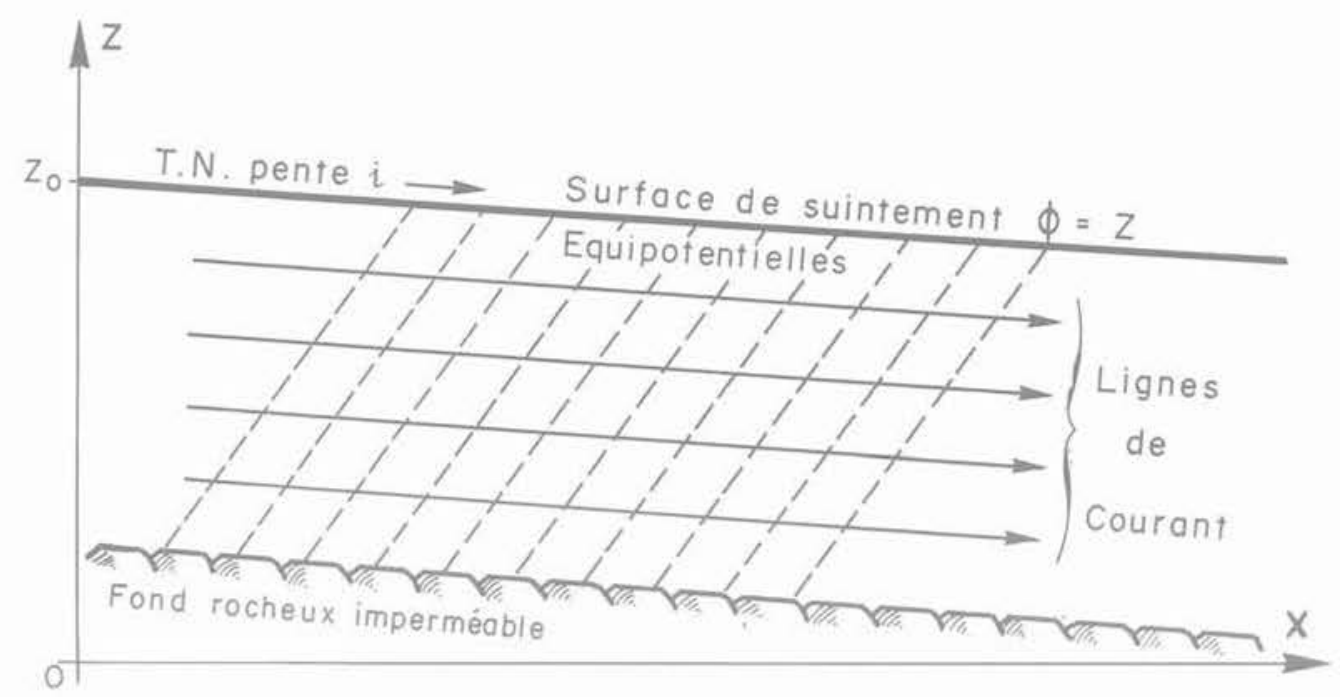

- Potentiel avec gradient vertical iv:

$$
\phi=i_{v} \cdot z-i \cdot\left(1-i_{v}\right) \cdot x+z_{o} \cdot\left(1-i_{v}\right)
$$

- Equitibre avec la pente: conditions sur les vitesses de filtration

$$
\frac{V_{V}}{V_{H}}=-i
$$

- Permeabilités principales : horizontales $K_{H}$ et verticale $K_{V}$

$$
\left.\frac{K_{H}}{K_{v}}=\frac{i_{v}}{i^{2}} \cdot \frac{1}{\left(1-i_{v}\right.}\right)
$$

Fig. 6. - Aquifère anisotrope avec écoulement uniforme, en équilibre avec la pente. 


\subsubsection{L'image fournie par les données avant tra-} vaux

- Les données avant travaux indiquent une forte perméabilité. Des études antérieures avaient confirmé, par un bilan de l'aquifère, la valeur moyenne proche de $2 \times 10^{-3} \mathrm{~m} / \mathrm{s}$ obtenue par de nombreux essais Lefranc en sondage.

La nappe, affleurante, présente un gradient vertical régulier et dirigé vers le haut, qui ne peut guère s'expliquer par les alimentations connues. Globalement, la nappe. semble en équilibre avec la rivière.

Une telle disposition peut être décrite simplement par un écoulement anisotrope uniforme, en équilibre avec la pente (fig. 6).

La pente de ces dépôts fluvioglaciaires étant faible, on peut admettre que les directions principales de perméabilité sont l'horizontale et la verticale.

Dans ces hypothèses, l'analyse des perturbations piézométriques apportées par un ruisseau à l'aval immédiat du barrage fournit un encadrement de l'anisotropie :

$$
60 \leq \frac{\mathrm{kH}}{\mathrm{kv}} \leq 200
$$

- L'analyse des mesures de perméabilité indique une structure particulière (fig. 7);

- une couche superficielle plutôt perméable,

- un interface, localisée entre 15 et 20 m de profondeur par les mesures de perméabilité dans l'axe de la future paroi, et détecté à $17 \mathrm{~m}$ par des mesures piézométriques dans l'axe du barrage, $100 \mathrm{~m}$ à l'aval de la paroi,

- sous l'interface, un entrecroisement de zones perméables et de zones moins perméables, d'une géométrie difficile à préciser.

- Au moyen d'un modèle d'écoulement utilisant les éléments finis, il apparaît alors que les écoulements naturels peuvent être fidèlement représentés (fig. 8) par un aquifère à perméabilité horizontale uniforme $\left(2,5 \times 10^{-3} \mathrm{~m} / \mathrm{s}\right)$, comprenant deux couches à fort contraste d'anisotropie (10 et 150) et dont l'interface se situe à $17 \mathrm{~m}$ de profondeur ;

- La géométrie de l'aquifère et de la fenêtre sous paroi (fig. 7) introduit des effets transversaux dans l'écoulement lorsque la paroi est terminée. De ce fait, le modèle plan vertical mis au point précédemment ne
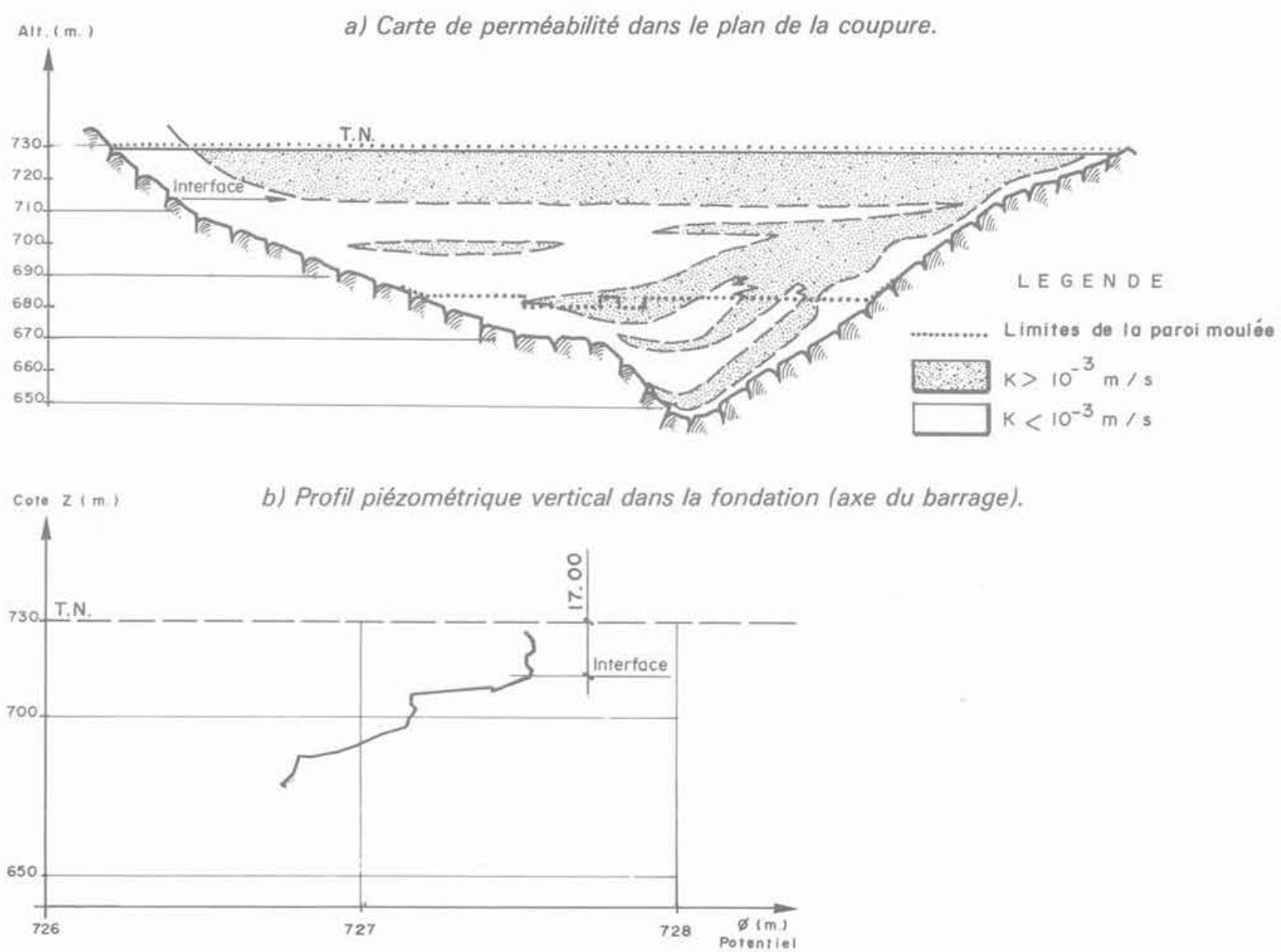

Fig. 7. - Interprétation hydrogéologique. 


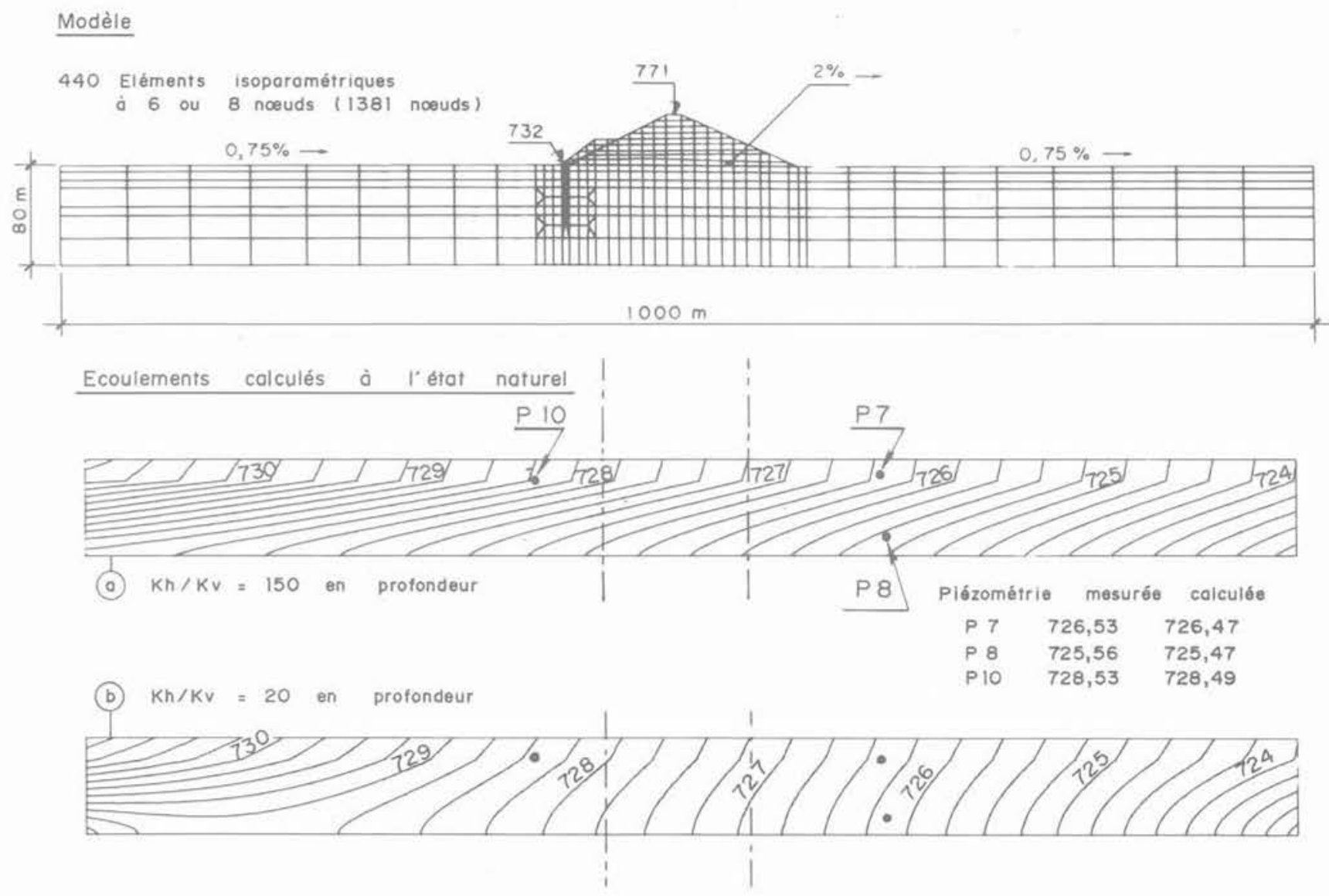

Fig. 8. - Modélisation des écoulements sous le barrage.

permet pas de calculer directement le débit avec la coupure incomplète.

Deux méthodes permettent d'évaluer le débit transitant dans l'aquifère, à partir de modèles plans verticaux, l'une par excès, l'autre par défaut (fig. 9).

- La méthode d'intégration transversale représente l'écoulement réel par une série de modèles plans verticaux correspondant à autant de coupes en long dans l'aquifère. Cette méthode qui introduit un cloisonnement vertical étanche en long dans l'écoulement, ne permet pas l'équilibrage transversal de la nappe au voisinage de la fenêtre sous paroi, et le débit qui y transite est donc calculé par défaut.

- La méthode d'intégration verticale consiste à remplacer les strates de l'aquifère, de largeur variable avec la cote, par des strates fictives de largeur constante mais de même transmissivité que les strates réelles correspondantes. Pratiquement cette méthode consiste à corriger les perméabilités des strates du modèle par un facteur géométrique fonction de la cote, et le débit est obtenu par simple intégration verticale des débits unitaires calculés dans ce modèle plan "corrigé ".

Cette méthode permet l'équilibrage transversal, mais ne tient pas compte des pertes de charge associées à la convergence et divergence transversale des lignes de courant au voisinage de la fenêtre sous paroi, le débit transitant sous la paroi est donc estimé par excès.

Ces deux méthodes donnent le même résultat, théoriquement exact, d'une part pour un aquifère en équilibre avec la pente, sans coupure, prismatique et de section quelconque, et d'autre part pour un aquifère avec coupure incomplète, lorsque la section en travers est un rectangle à fond horizontal.

Dans le cas de la vallée du Verney, de section triangulaire, un calcul test avec la paroi moulée et la pression de la retenue normale à l'amont, indique que l'écart relatif entre les débits sous paroi calculés par ces deux méthodes est de $13 \%$. Cet écart, non significatif si l'on tient compte de l'imprécision qui règne par ailleurs sur les divers paramètres, justifie l'emploi indifférent de l'une ou l'autre de ces deux méthodes.

La seconde, plus rapide, a été utilisée systématiquement.

\subsubsection{Contrôle par les mesures réalisées durant} travaux

A la fermeture de la coupure, un rabattement se produit à l'aval, dont la vitesse peut être tirée des mesures piézométriques d'une part, et d'autre part calculée au moyen du modèle d'écoulement. 
a) Méthode d'intégration transversale.

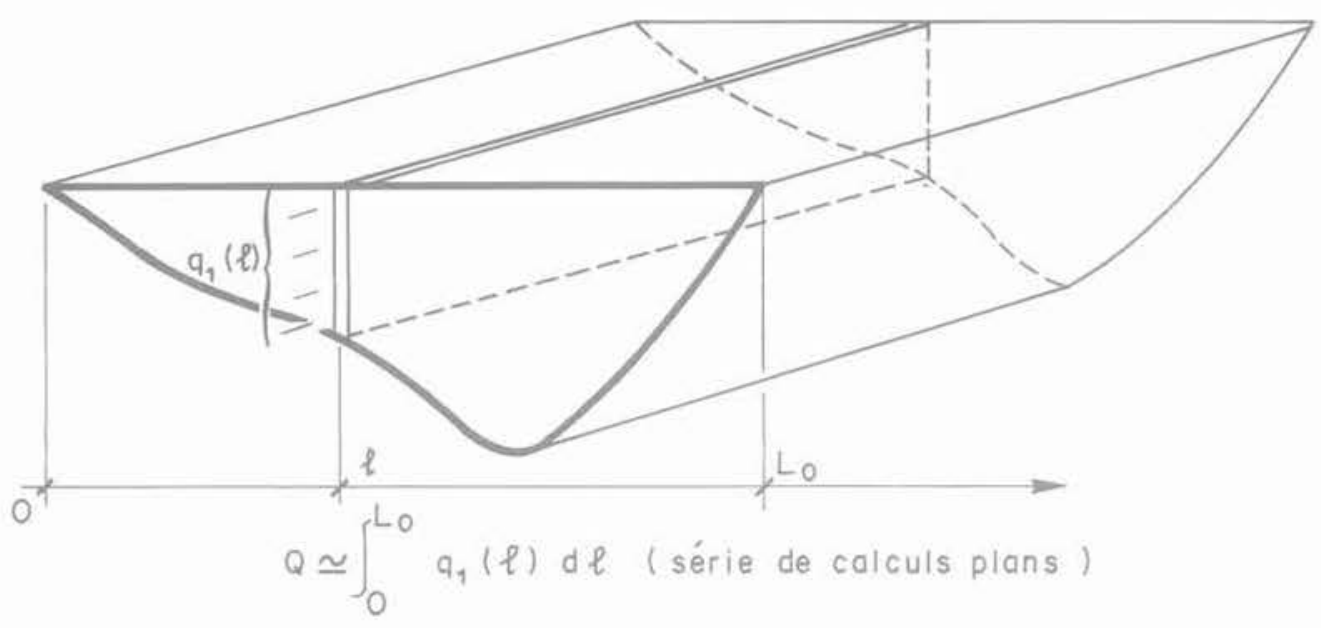

b) Méthode d'intégration verticale.
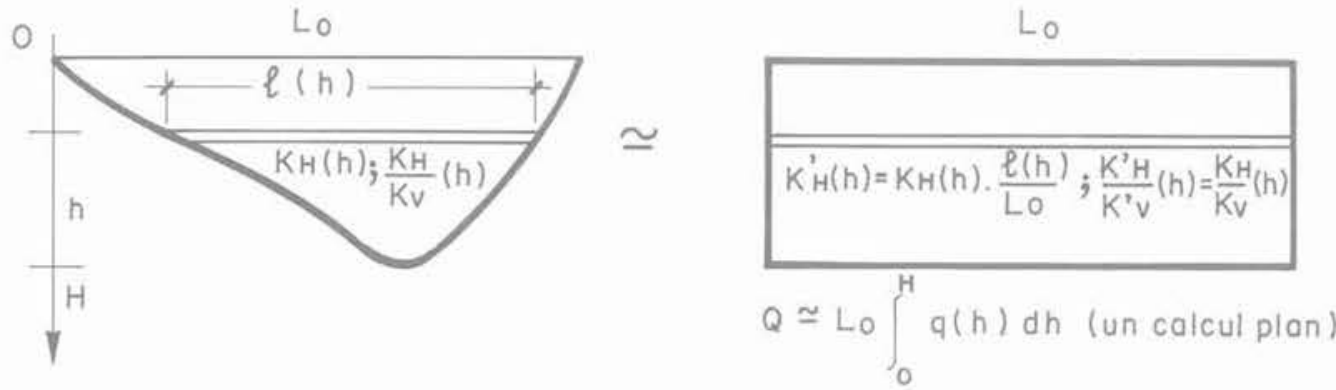

Fig. 9. - Evvaluation du débit de percolation.

- Les mesures piézométriques indiquent (fig. 10a) lors de la fermeture de la paroi moulée, un rabattement aval de 2 à $3 \mathrm{~m}$ qui se poursuit régulièrement sur plusieurs mois. A l'amont, apparaît un relèvement de quelques décimètres et l'équilibre y est atteint plus rapidement qu'à l'aval. Avant la fermeture, la paroi partiellement réalisée provoque déjà un certain rabattement préalable. Les mesures piézométriques montrent que ce rabattement préalable est de l'ordre de $20 \%$ du rabattement total, et que l'écoulement dans la nappe à l'amont et à l'aval est encore proche des conditions piézométriques de l'écoulement naturel avant travaux de fermeture.

La piézométrie superficielle étant vraisemblablement reliée au niveau de la rivière, la vitesse de rabattement "observée» sur l'aval, a été calculée sur les données piézométriques diminuées du niveau de l'Eau d'Olle. afin de s'affranchir de l'effet des fluctuations de la rivière.

- Dans le modèle d'écoulement, on peut calculer les conséquences de l'apparition aimmédiate » de la coupure dans l'écoulement naturel (fig. 10b).

Cette schématisation peut apparaître un peu brutale au vu des conditions réelles de fermeture progressive de la paroi moulée, elle est cependant tout à fait corroborée par les observations faites sur les mesures piézométriques.

Dans le modèle d'écoulement soumis à de telles conditions, apparaît un fort déficit en alimentation verticale en surface à l'aval de la coupure. Ce déficit est proportionnel à la vitesse de descente de la nappe, et fonction de l'indice des vides du terrain.

Pour un indice des vides compris entre 0,3 et 0,4 , correspondant à la densité en place de ces matériaux à granulométrie étalée, on obtient ainsi une fourchette de vitesses de rabattement «calculées .

La comparaison des situations observées et calculées à la fermeture de la paroi (tableau I), montre que le modèle d'écoulement calé sur les conditions naturelles donne encore une représentation fidèle de cet écoulement à la fermeture, assez éloigné des conditions régulières de l'écoulement naturel.

A l'amont le modèle d'écoulement indique qu'un débit notable est rejeté en surface, loin de la coupure, et donc vraisemblablement retourné à la rivière dans la réalité. 
Tableau I

Comparaison entre les situations observées et calculées à la fermeture

\begin{tabular}{|l|c|c|c|}
\hline \multicolumn{2}{|c|}{ VALEURS OBSERVEES } & \multicolumn{2}{c|}{ VALEURS CALCULÉES } \\
\hline & & \multicolumn{1}{c|}{ Anisotropie 10 entre 0 et $17 \mathrm{~m}$ de profondeur } \\
\hline & $\begin{array}{c}\text { Anisotropie } 150 \\
\text { au-dessous de } 17 \mathrm{~m}\end{array}$ & $\begin{array}{c}\text { Anisotropie } 20 \\
\text { au-dessous de } 17 \mathrm{~m}\end{array}$ \\
\hline $\begin{array}{l}\text { Vitesse de rabattement } 220 \mathrm{~m} \text { à l'aval } \\
\text { de la paroi }(\mathrm{m} / \mathrm{s} \text { ) }\end{array}$ & $12 \times 10^{-7}$ & 11 à $13,4 \times 10^{-7}$ & 0 à $3 \times 10^{-7}$ \\
\hline $\begin{array}{l}\text { Vitesse de rabattement } 330 \mathrm{~m} \text { à l'aval } \\
\text { de la paroi (m/s) }\end{array}$ & $11 \times 10^{-7}$ & 4 à $5 \times 10^{-7}$ & 0 \\
\hline $\begin{array}{l}\text { Gradient piézométrique vertical ; } \\
220 \mathrm{~m} \text { à l'aval de la paroi }\end{array}$ & $0,6 \%$ & $0,5 \%$ & 0 \\
\hline
\end{tabular}

a) Evolution de la piézométrie

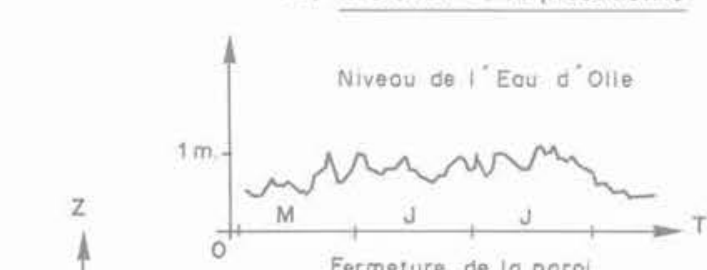

b) Schéma des conditions aux limites
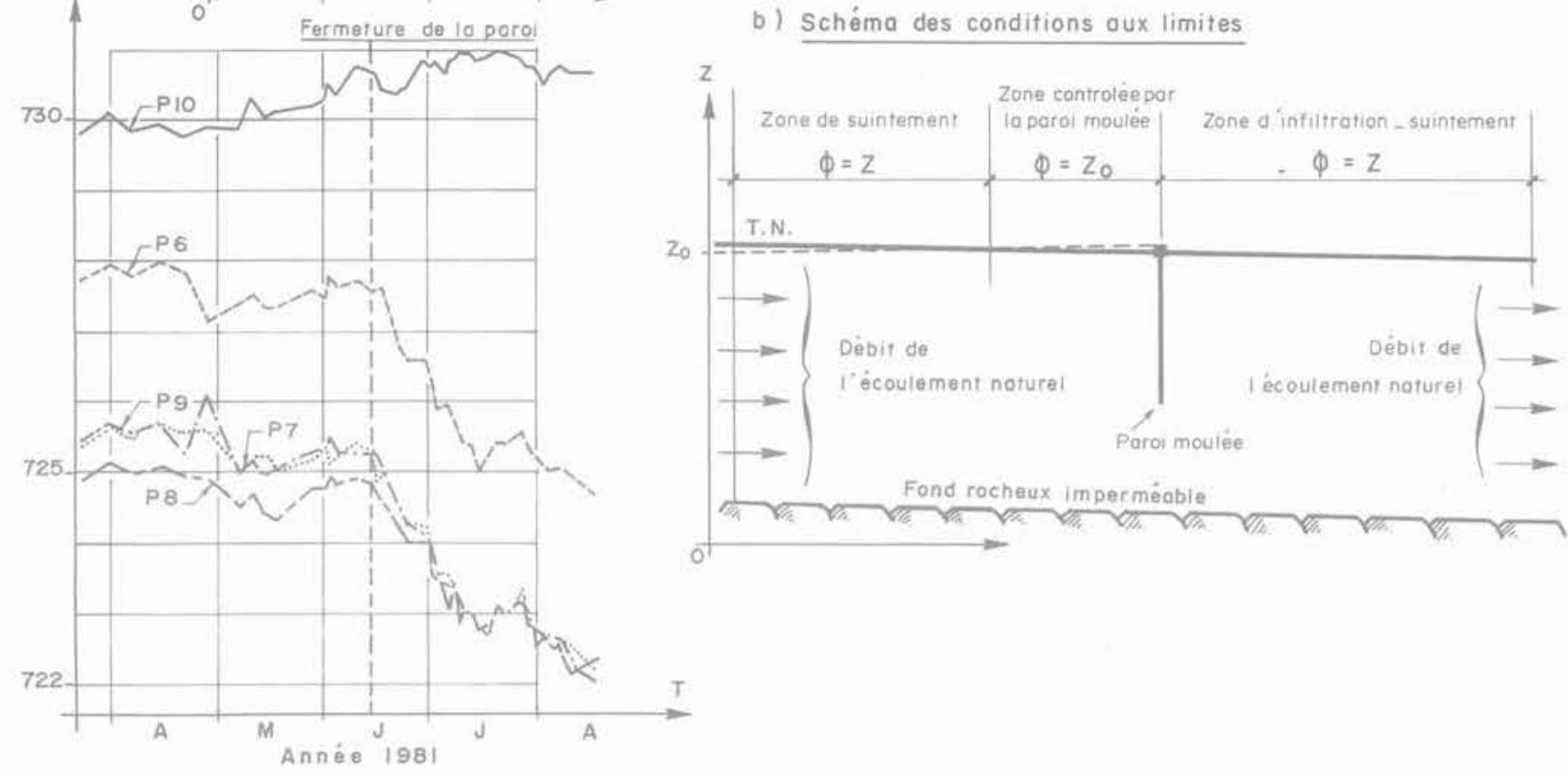

Fig. 10. - Fermeture de la paroi moulée.

A proximité immédiate de la coupure, le débit rejeté en surface dans l'espace compris entre le batardeau et la paroi moulée est calculé à quelques $1 / \mathrm{s}$, ce qui correspond tout à fait au débit observé dans l'exutoire provisoire installé en tête de paroi moulée lors des travaux de fermeture.
2.1.3. L'exploitation du modèle hydrogéologique Afin, d'une part, de vérifier que la coupure incomplète de la fondation par la paroi moulée satisfaisait effectivement les objectifs du projet, et d'autre part de préciser les écoulements sous le barrage, les conditions de fonctionnement normal ont été simulées (fig. 11). 


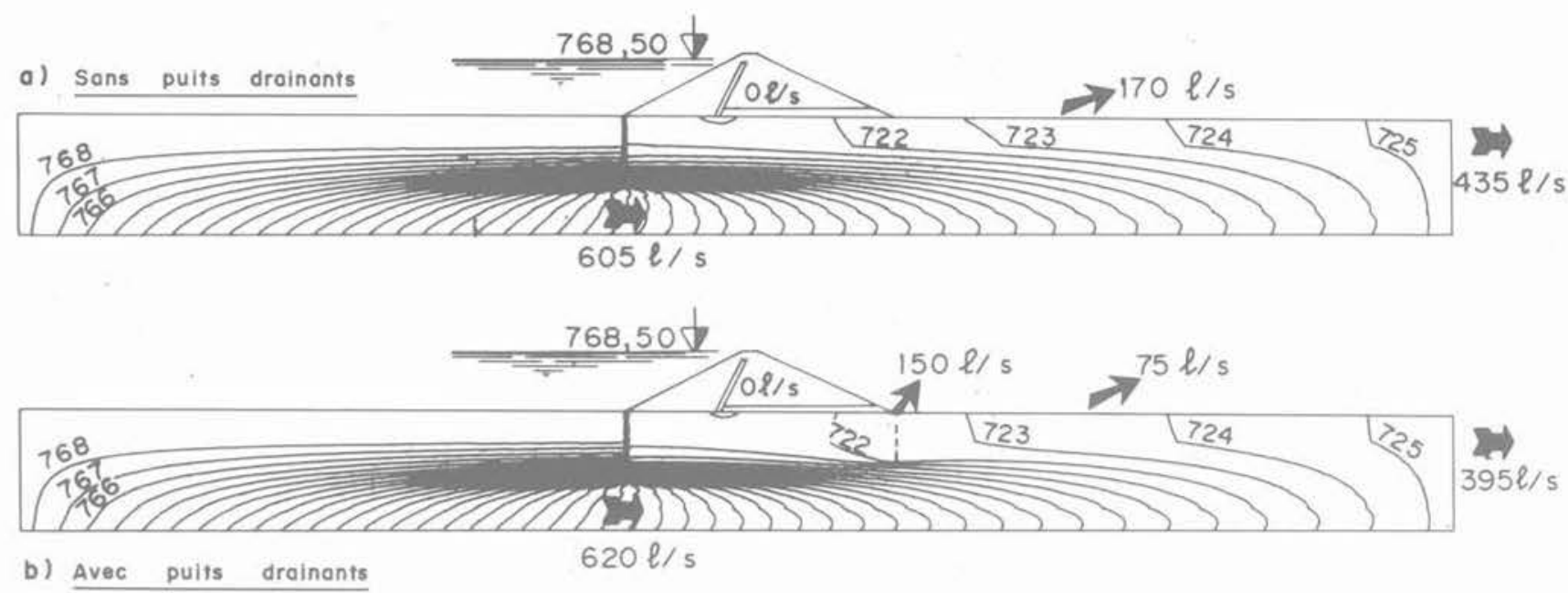

Fig. 11. - Ecoulements sous le barrage avec la retenue normale.

Les résultats essentiels sont :

- aucun écoulement en provenance de la fondation ne percole dans le tapis drainant du barrage, en fonctionnement normal. La nappe n'atteint donc pas ce tapis drainant. Une surface libre s'établira dans la fondation à l'aval de la paroi sous le barrage et oscillera avec le marnage;

- les débits qui passent sous la paroi moulée varient entre $200 \mathrm{l}$ /s pour la cote minimale d'exploitation, soit sensiblement le débit de l'état naturel, et $600 \mathrm{l} / \mathrm{s}$ pour la cote maximale d'exploitation.

\subsection{Analyse des mouvements dans la fondation durant les travaux}

La construction des remblais a été menée à un rythme rapide au début de l'année 1982, l'essentiel des remblais a été mis en place en moins de trois mois. Le procédé de construction a nécessité la réalisation d'une piste de chantier, située en hors profil sur le parement amont, et enlevée par la suite. Durant la période de construction, les relevés systématiques des appareils d'auscultation ont fourni un flot régulier d'informations sur l'évolution de la fondation : outre les piézomètres et cellules de pression dont les résultats sont analysés plus haut, le clinomètre et les extensomètres en forage ainsi que le furet hydraulique ont permis un suivi détaillé des mouvements sous le barrage.

Dans un premier temps, pour cerner les principaux caractères mécaniques de la fondation, il a été choisi d'analyser en détail deux étapes significatives de cette construction.

\subsubsection{Mouvements et déformations mesurés} La figure 12 détaille les mouvements et déformations observées lors de la mise en place de deux tranches de remblai successives de $12 \mathrm{~m}$ puis $14 \mathrm{~m}$ d'épaisseur.

- Dans l'axe du barrage, la fondation soumise à des efforts essentiellement verticaux présente des mouvements verticaux notables. (Ces mouvements, très largement supérieurs aux valeurs attendues qui avaient guidé le choix de la course des appareils d'extensométrie, ont progressivement mis hors service les capteurs profonds de l'extensomètre axial.)

Ces mouvements sont de l'ordre de 2 à $3 \mathrm{~cm}$ en tassement à la surface de la fondation pour chaque mètre de remblai mis en place. Ils sont dus pour l'essentiel au tassement des niveaux intermédiaires de la fondation. Ils se concentrent particulièrement entre 18 et $33 \mathrm{~m}$ de profondeur sous le barrage, où la déformation verticale cumulée atteint $2,5 \%$ pour la mise en place de ces $26 \mathrm{~m}$ de remblai.

- Au pied amont du barrage, la fondation soumise à des efforts obliques enregistre des déformations de cisaillement notables, détectées par le clinomètre.

L'intégration de ces déformations représente un mouvement vers l'amont de $12 \mathrm{~cm}$ au niveau T.N. $-18 \mathrm{~m}$, pour la mise en place de ces $26 \mathrm{~m}$ de remblai. Ces déformations de cisaillement sont concentrées dans les niveaux intermédiaires de la fondation, et en particulier, tout comme sous l'axe du barrage, dans le niveau situé entre 18 et $33 \mathrm{~m}$ de profondeur. Dans ce niveau, sous l'effet de la mise en place des $26 \mathrm{~m}$ de remblai, la déformation de cisaillement enregistrée atteint $0,6 \%$, la déformation verticale ne dépasse pas cette valeur.

\subsubsection{Interprétation par l'Élasticité}

- Afin de préciser quantitativement le contraste de déformabilité ainsi mis à jour, il a été décidé de réaliser une analyse exhaustive des caractéristiques élastiques apparentes dans chaque couche de fondation située entre deux niveaux de capteurs de déformation. 


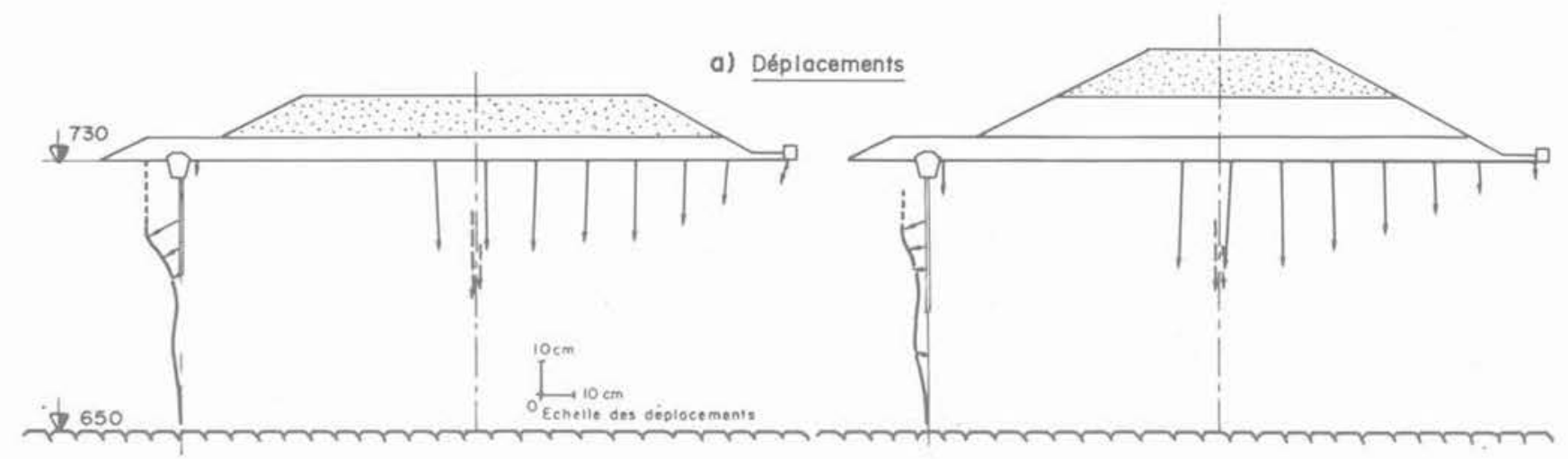

b) Déformotions

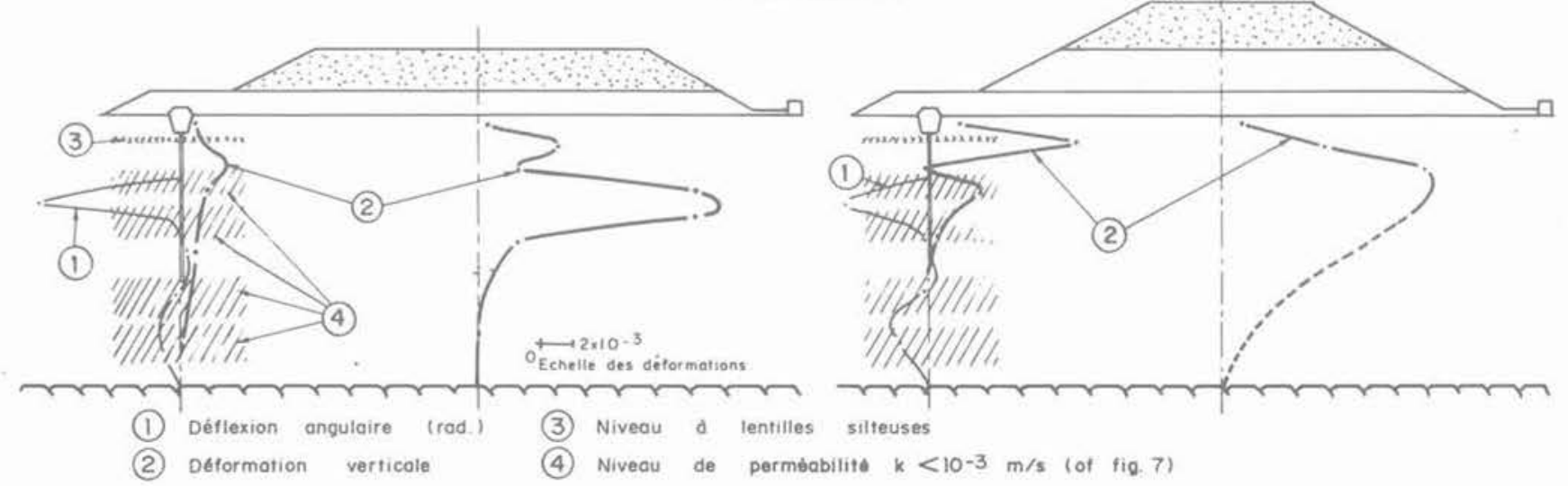

Fig. 12. - Mouvements dans la fondation durant la construction du barrage.

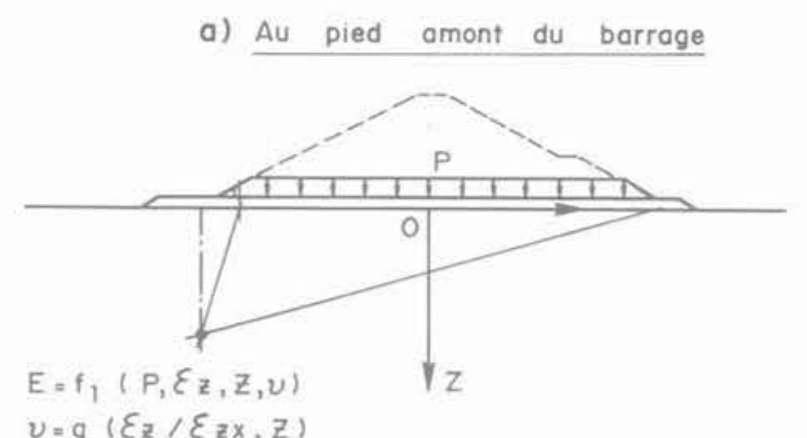

b) Dans l'axe du barrage

c) Par cobèrence entre o) et b)

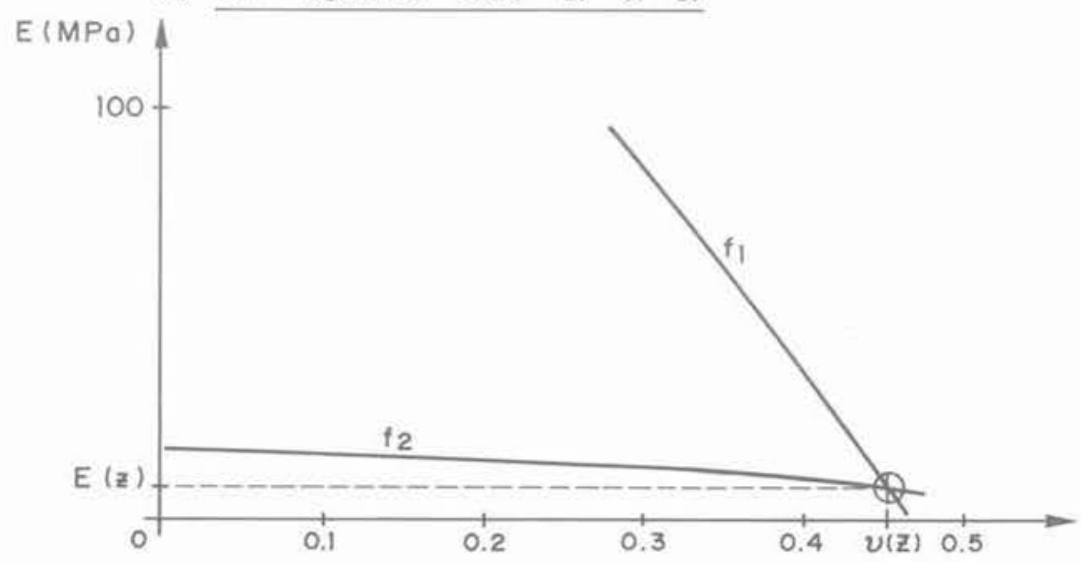

Fig. 13. - Détermination de coefficients élastiques apparents dans la fondation. 


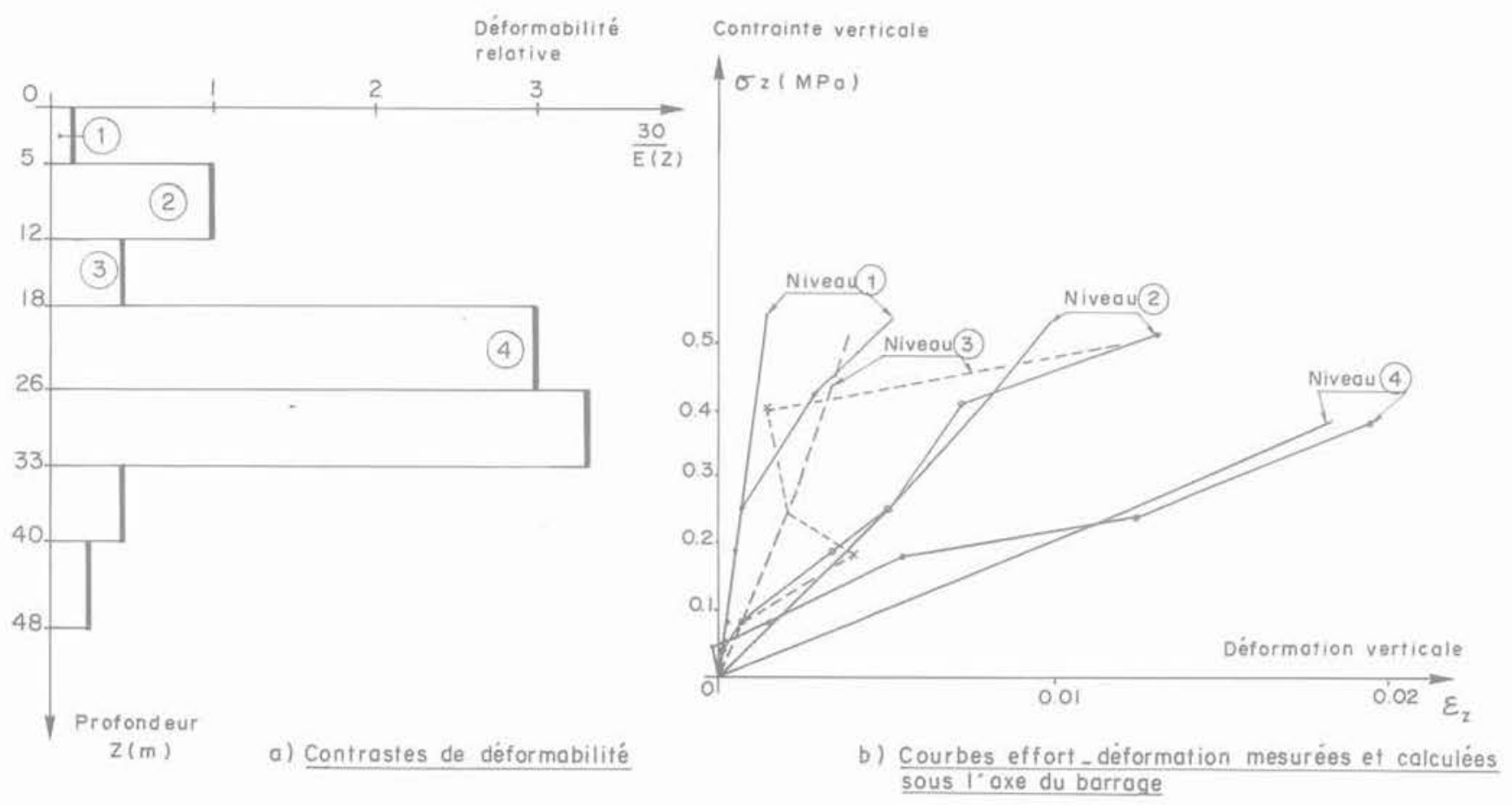

Fig. 14. - Contrastes de déformabilité et non-linéarité mécanique dans la fondation.

La présence d'un même niveau particulièrement déformable dans l'axe du barrage et au voisinage de son pied amont confortait l'hypothèse d'une stratification horizontale de la fondation. Cette hypothèse avait déjà été suggérée par l'anisotropie élevée trouvée dans l'analyse hydrogéologique.

Par ailleurs, le toit de ce niveau déformable correspondait précisément à l'interface hydrogéologique localisé vers $17 \mathrm{~m}$ de profondeur.

- Dans cette hypothèse de fondation stratifiée, les mesures de déformation permettaient la détermination, ainsi qu'un certain contrôle, des caractéristiques élastiques apparentes de chaque couche.
La méthode employée est celle de Steinbrenner (POULOS, DAVIS, 1974), qui consiste à supposer que la distribution des contraintes dues à la mise en place approximativement la même dans la fondation stratifiée qu'avec une fondation homogène semi-infinie.

Pour une étape de chargement donnée, connaissant les contraintes calculées par la méthode de Steinbrenner et les déformations mesurées par les extensomètres, on peut alors déduire des caractéristiques élastiques apparentes locales par les méthodes analytiques de l'Élasticité (fig. 13).

- Les résultats complets (tableau II) indiquent alors. outre le détail du contraste de déformabilité (fig. 14a),

Tableau II

Coefficients élastiques apparents dans la fondation

\begin{tabular}{|c|c|c|}
\hline $\begin{array}{c}\text { PROFONDEUR } \\
\text { SOUS LE BARRAGE }\end{array}$ & $\begin{array}{c}\text { MODULE D'YOUNG } \\
\text { (MPa) }\end{array}$ & $\begin{array}{c}\text { COEFFICIENT } \\
\text { DE POISSON }\end{array}$ \\
\hline 0 à $5 \mathrm{~m}$ & 230 & 0,3 \\
5 à $12 \mathrm{~m}$ & 30 & 0,3 \\
12 à $18 \mathrm{~m}$ & 70 & 0,33 \\
18 à $26 \mathrm{~m}$ & 10 & 0,43 \\
26 à $33 \mathrm{~m}$ & 9 & 0,45 \\
33 à $40 \mathrm{~m}$ & 70 & 0,3 \\
40 à $48 \mathrm{~m}$ & 130 & 0,3 \\
\hline
\end{tabular}


que le niveau particulièrement déformable entre 18 et $33 \mathrm{~m}$ de profondeur semble s'être déformé essentiellent en cisaillement, pratiquement sans réduction de volume. En effet, la valeur élevée de son coefficient de Poisson apparent, obtenu et contrôlé par la méthode de la figure 13 , correspond à un matériau pratiquement incompressible.

Aucune élévation substantielle des pressions interstitielles dans la fondation n'a cependant été notée au voisinage de ce niveau. Selon une interprétation émise alors, ce niveau pourrait consister en intercalations rapprochées de lentilles minces subhorizontales de matériaux plutôt fins et peu perméables avec des lentilles de matériaux plus drainants.

Une telle structure est corroborée par la valeur élevée de l'anisotropie globale trouvée en perméabilité (150) et par la valeur élevée de la perméabilité horizontale moyenne $\left(2,5 \times 10^{-3} \mathrm{~m} / \mathrm{s}\right)$.

- L'exploitation de ces caractéristiques élastiques apparentes permet également de comparer, dans l'axe du barrage, les courbes effort-déformations calculées à celles qui ont été observées (fig. 14b).

Une certaine non-linéarité apparaît clairement.

\subsection{Analyse des conséquences sur la paroi moulée}

La présence inopinée de ce niveau déformable avait certainement amplifié les déformations de cisaillement prévues au voisinage du pied amont du barrage, à l'emplacement de la paroi moulée.

Afin de vérifier que la paroi moulée en béton plastique avait supporté sans dommage irréversible de telles déformations, imposées par la fondation, une étude particulière a alors été engagée.

Dans une première étape, la rhéologie du béton plastique du Verney a été étudiée à partir d'essais de laboratoire, afin de définir un domaine de réversibilité mécanique.

Dans une deuxième étape, la position de l'état de contraintes évalué dans la paroi a été comparé à la limite du domaine de réversibilité du béton plastique.

\subsubsection{Rhéologie du béton plastique du Verney}

- Le béton de la paroi moulée a été fabriqué par malaxage de sable, de gravier, et d'un coulis d'argileciment. Deux séries d'essais triaxiaux drainés ont été réalisées sur ce béton par le laboratoire de Mécanique des Sols de l'École Centrale:

- une première série réalisée entre 29 et 47 jours après la fabrication des éprouvettes;

- une deuxième série, réalisée entre 190 et 223 jours après la fabrication.

- Les variations de volume ayant été mesurées systématiquement au cours des essais, l'ensemble des don- nées a été exploité par une méthode synthétique, celle des diagrammes de dilatance (fig. 15).

Cette méthode consiste à porter les résultats de l'essai dans un plan dont les coordonnées sont :

- en abscisses le rapport des vitesses de déformation principales;

- en ordonnées le rapport des contraintes principales effectives.

Par rapport aux courbes d'essai usuelles, ces diagrammes font apparaître plus clairement les différences de comportement :

- l'élasticité linéaire se traduit par une droite verticale dont l'abscisse est précisément le coefficient de Poisson;

- la plasticité parfaite à volume constant se traduit par un point d'abscisse $1 / 2$;

- un comportement granulaire dilatant (grains ou blocs rigides en contact frictionnel) se traduit par une droite inclinée passant par l'origine, et dont la pente est en relation directe avec la friction entre les grains.

- Schématiquement, les diagrammes de dilatance de la figure 16 montrent :

- en début d'essai, une forte compressibilité apparente, indice d'une phase de serrage;

- après cette phase de serrage, la portion de courbe pratiquement verticale correspond au domaine élastique :

- au-delà, la contraction élastique (reliée au coefficient de Poisson) est graduellement oblitérée par la dilatation due à la propagation de fissures dans le béton plastique, et lorsque les fissures établies forment un réseau continu tel qu'il puisse y avoir séparation des morceaux, le comportement s'apparente à celui d'un matériau granulaire dilatant après le pic;

- l'ampleur de cette phase de comportement granulaire dilatant décroît nettement avec le confinement, par un effet d'épaufrement des morceaux de béton plastique:

- dans le domaine exploré en contraintes et déformations par ces essais, on ne décèle aucun effet d'effondrement de structure ni de comportement plastique à volume constant.

Les valeurs moyennes des constantes élastiques sont :

$$
\begin{array}{ll}
\text { Module de Young } & E \approx 230 \mathrm{MPa} \\
\text { Coefficient de Poisson } & v \approx 0,22
\end{array}
$$

- Au cours d'une sollicitation, l'apparition d'irréversibilités est liếe à la rupture de liaisons du ciment dans le coulis et à la propagation de fissures conséquentes à ces ruptures. Les effets de serrage constatés lors des essais triaxiaux suggèrent d'ailleurs la préexistence d'une certaine microfissuration, peut être liée aux effets de la prise dans le béton. 

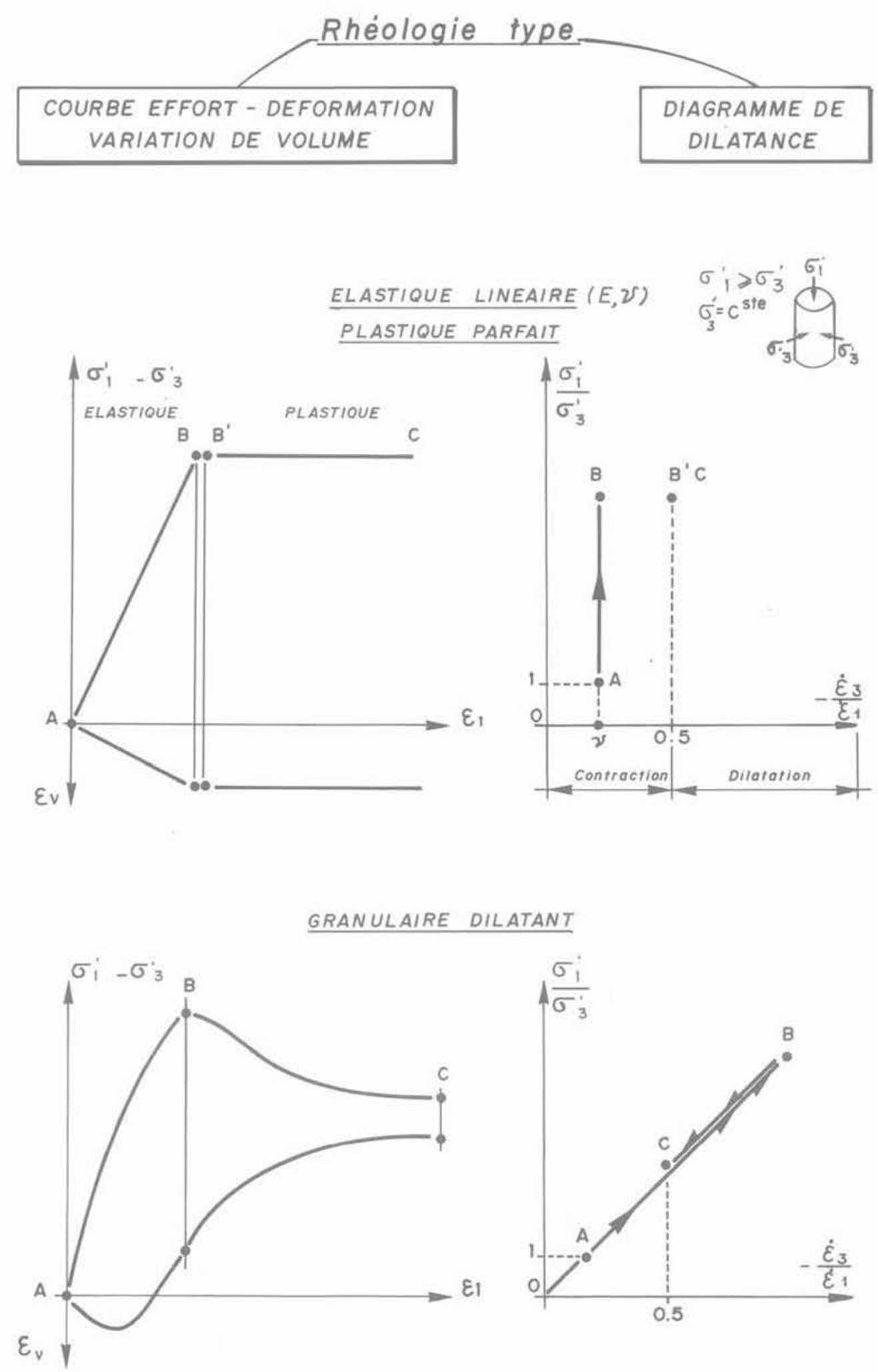

Fig. 15. - Interprétation par les diagrammes de dilatance de la rhéologie mesurée à l'essai triaxial drainé. 
a) Courbes d'essai-Série A

b) Diagrammes de dilatance

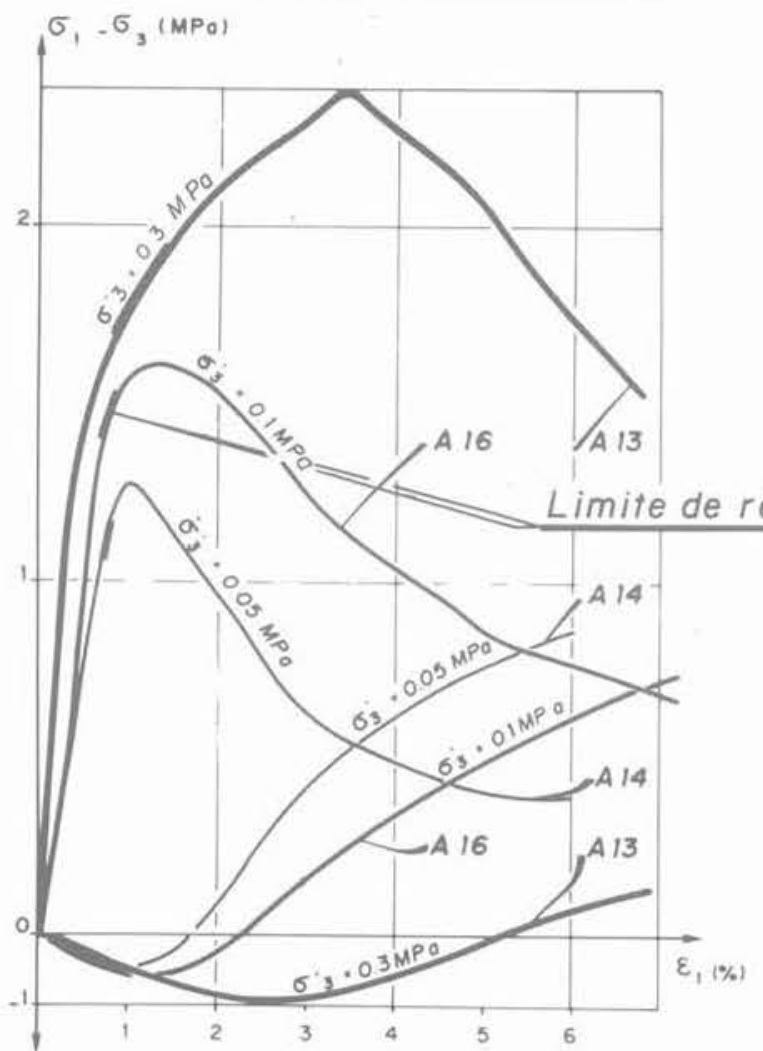

$\varepsilon_{v}(\%)$

c) Courbes d'essai-Série B

$$
\sigma_{1}, \sigma_{3}(\mathrm{MPa})
$$

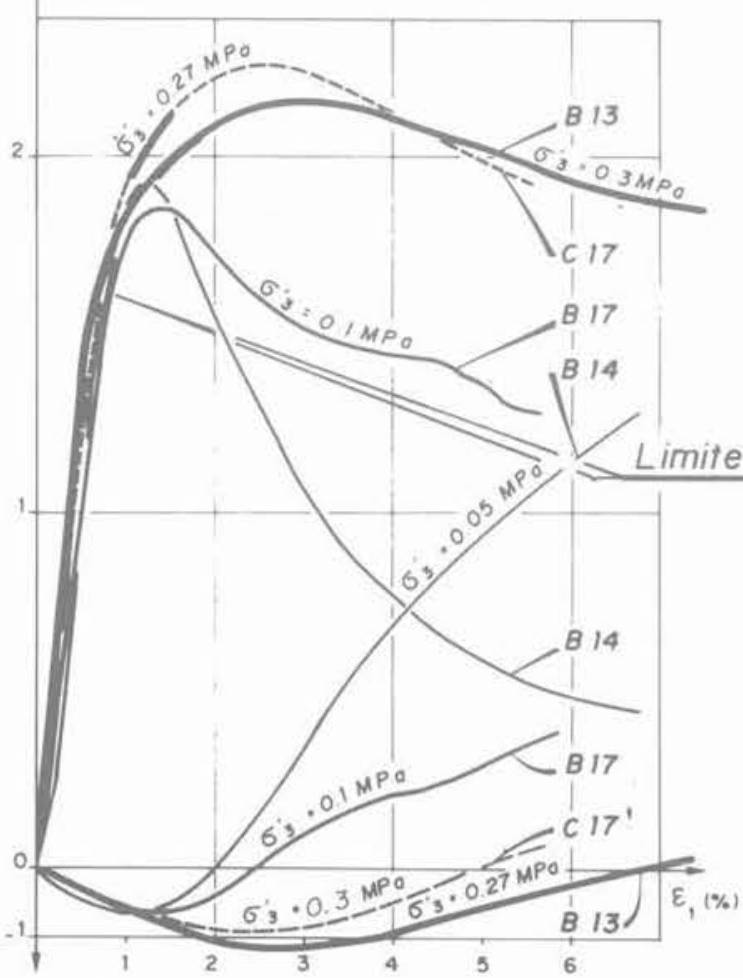

$\varepsilon_{v}(\%)$

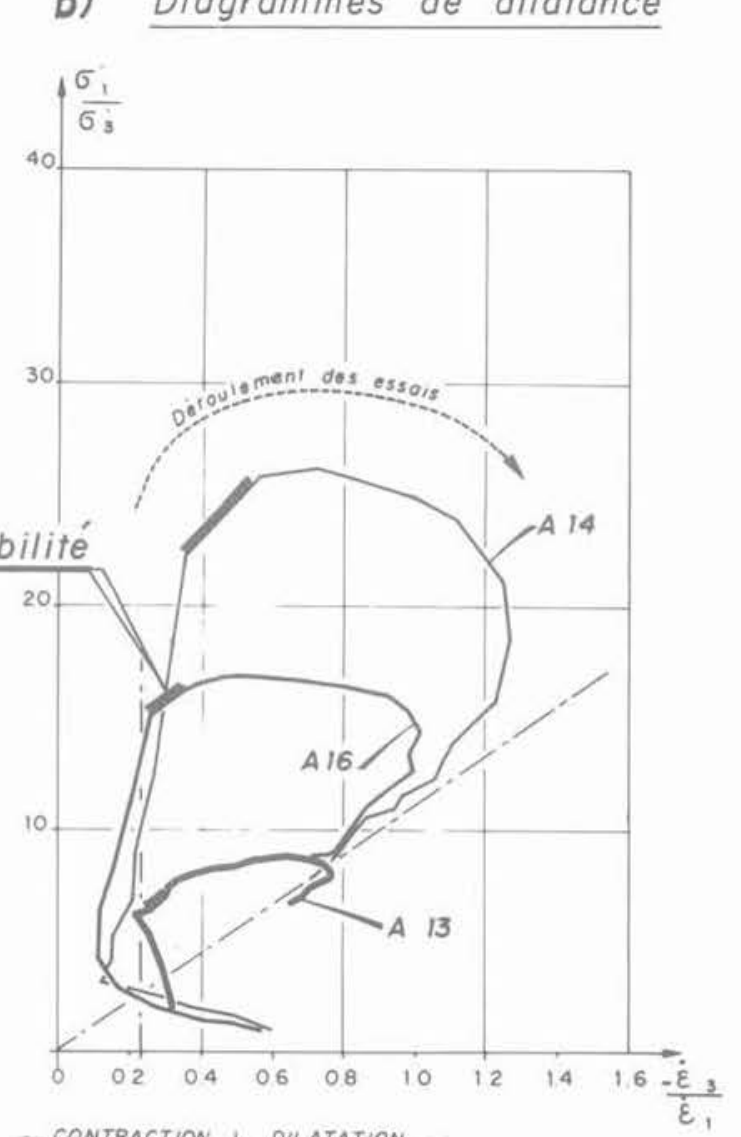

- CONTRACTION + DILATATION -

d) Diagrammes de dilatance

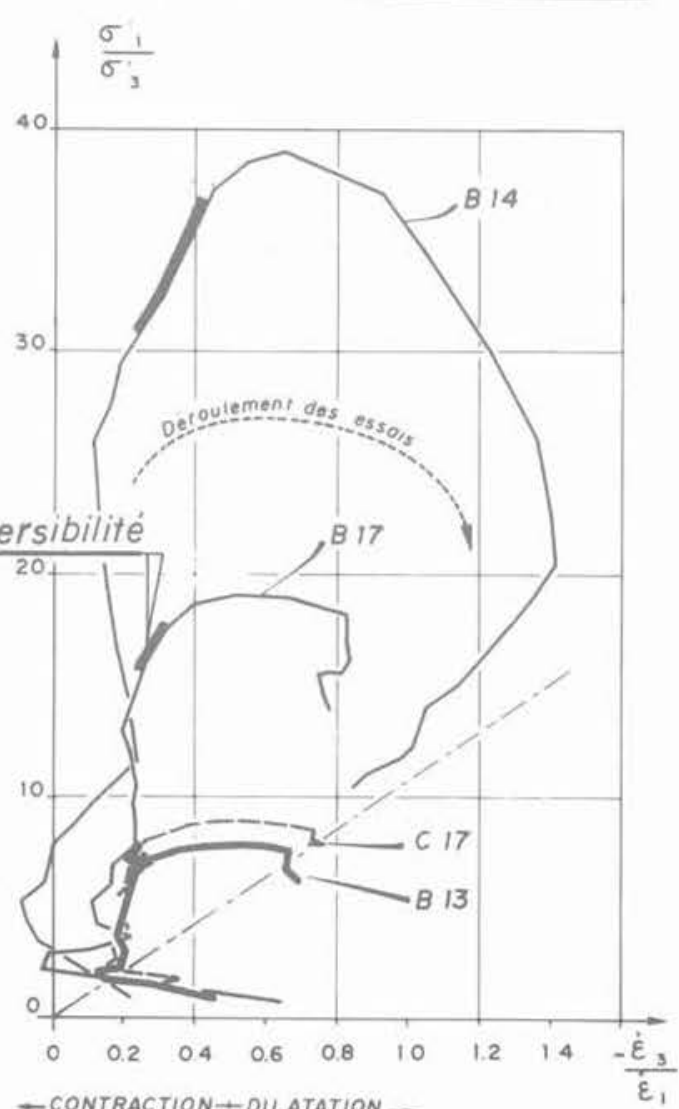

- CONTRACTION t OILATATION

Fig. 16. - Béton plastique du Verney - Essais triaxiaux. 
Au voisinage de sa limite de réversibilité, le comportement de ce béton d'argile-ciment serait donc celui d'un matériau microfissuré.

Un modèle simple existe pour ce type de matériaux, qui donne une interprétation convaincante des résultats expérimentaux sur les mortiers et bétons ainsi que sur les roches (LINO - 1980).

Ce modèle, fondé sur les mécanismes énergétiques qui régissent la propagation des fissures, donne une limite de réversibilité, en fonction des contraintes extrêmes, qui vaut ici :

$\sigma_{1}^{\prime}=\tan \left(\frac{\pi}{4}+\frac{\varnothing_{1}}{2}\right)\left[2 R_{1}+\sigma_{3}^{\prime} \tan \left(\frac{\pi}{4}+\frac{\varnothing_{1}}{2}\right)\right]\{1\}$

où $\mathrm{R}$, est la résistance à la traction

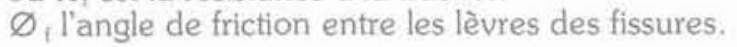

Dans l'équation $\{1\}$, le terme :

$$
2 R_{1} \cdot \tan \left(\frac{\pi}{4}+\frac{\varnothing_{1}}{2}\right)
$$

est voisin - théoriquement par défaut - de la résistance en compression simple.

Cette représentation à partir des contraintes principales extrêmes permet de passer des essais sous symétrie de révolution à une situation réellement tridimensionnelle.
L'évaluation de cette limite de réversibilité, réalisée sur les diagrammes de dilatance, est reportée sur les courbes d'essai figure 16 et détaillée figure 17.

\subsection{2. État de contraintes dans le béton de la paroi} - La paroi moulée avait certainement suivi les déformations de la fondation, concentrées dans le niveau entre 18 et $33 \mathrm{~m}$ de profondeur.

Dans un premier temps, l'investigation s'est donc limitée à ce niveau qui apparaissait comme un niveau de sollicitation maximale.

En première approximation, les déformations de la fibre moyenne de la paroi moulée ont été identifiées aux mesures données par le clinomètre et l'extensomètre situés une dizaine de mètres à l'aval.

- L'état de contraintes dans la paroi, dans une coupe amont-aval, correspond vraisemblablement à un état de déformation plane; il résulte de trois composantes:

- l'état de contrainte initial isotrope dû à la prise hydrostatique du béton:

- l'effet de compression amont-aval dû à la poussée des terres, joint à l'effort vertical dû à la déformation verticale;

- l'effet de flexion dû au gradient de déformations de cisaillement imposé par la fondation.

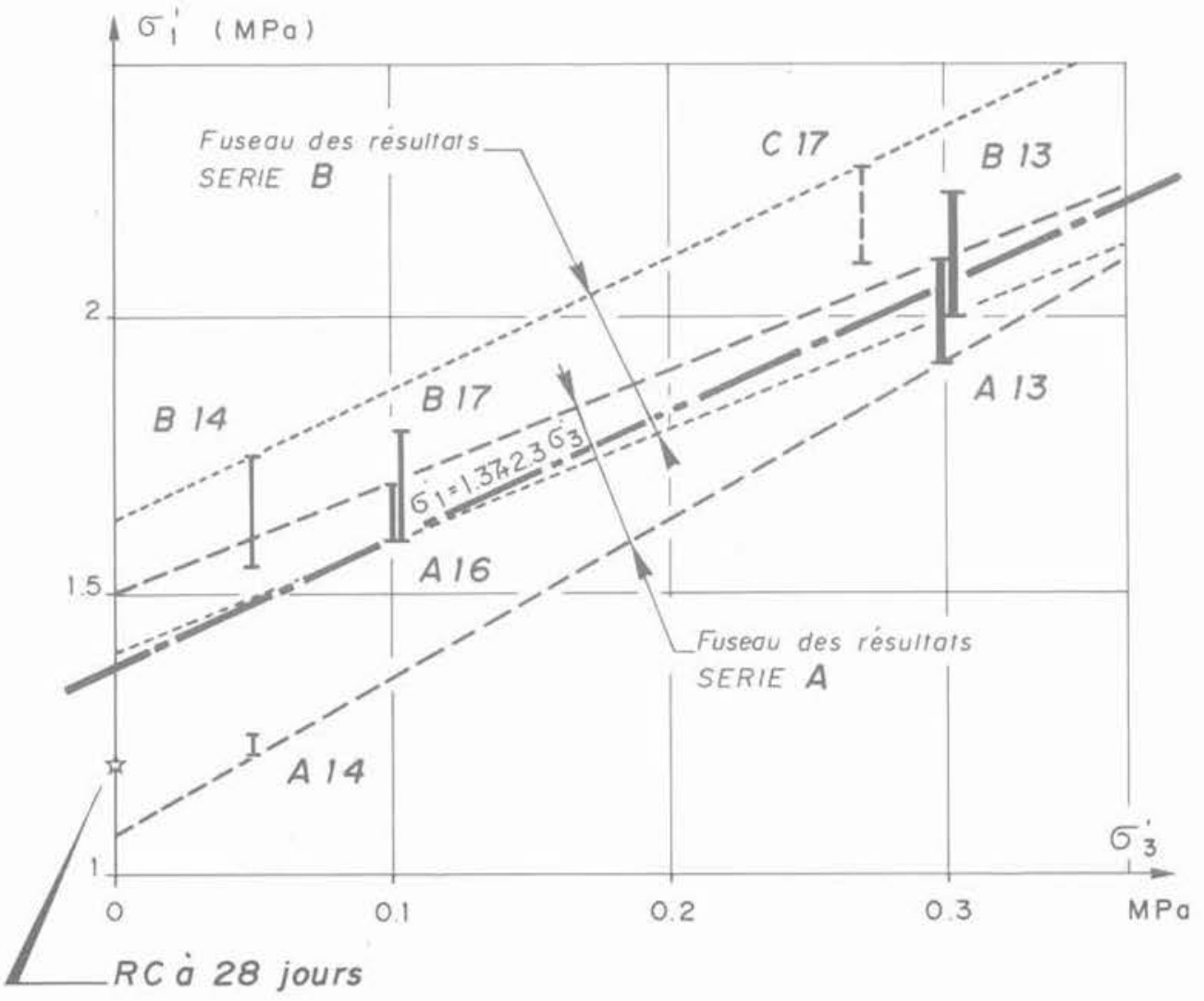

Fig. 17. - Béton plastique - Limite de réversibilité. 
Compte tenu de ce chargement, on peut admettre que les directions principales des contraintes, au voisinage des parements, sont sensiblement :

- la verticale;

- l'horizontale dans le sens amont-aval;

- l'horizontale dans le sens rive droite-rive gauche.

- Les méthodes de résistance des matériaux sur la flexion des plaques permettent d'évaluer les contraintes dans la paroi engendrées par les déformations. L'effet de la poussée des terres, estimé à partir de calculs réalisés au stade du projet, est faible en valeur relative.

Enfin, les mesures sur les cellules de pression au voisinage de la paroi permettent d'évaluer la pression interstitielle.

A la cote 703 , correspondant au milieu du niveau déformable dans la fondation, les contraintes calculées dans le béton de la paroi sont données au tableau III :

- On peut alors comparer les états de contraintes effectives déduits du tableau III à la limite de réversibilité du béton déterminée précédemment.

A contrainte mineure donnée, le degré de sollicitation peut être apprécié par l'intensité relative du déviateur des contraintes dans le béton par rapport au déviateur correspondant à la limite de réversibilité.

Dans ce sens, on trouve que :

- côté amont, le béton est sollicité à $47 \%$ de sa limite de réversibilité :

- côté aval, le béton est sollicité à $72 \%$ de sa limite de réversibilité.

\subsection{Conclusions sur l'interprétation des mesures en place}

- A ce stade, les analyses hydrogéologique et mécanique avaient montré :

- une fondation fortement anisotrope et clairement stratifiée, présentant une forte hétérogénéité mécanique due à la superposition de niveaux hautement contrastés en déformabilité
- un niveau particulièrement déformable, épais de $15 \mathrm{~m}$ environ, dont le toit apparaissait également comme interface hydrogéologique.

L'évaluation des caractéristiques élastiques apparentes dans la fondation faisait apparaître un module d'Young moyen environ quatre fois plus faible que celui évalué lors du projet.

Le comportement mécanique de la fondation apparaissait nettement non linéaire.

Enfin, l'étude des conséquences sur la paroi moulée montrait que le béton y avait été sérieusement sollicité, sans toutefois franchir clairement sa limite de réversibilité.

- Ces éléments rassemblés soulignaient l'utilité d'une prévision du comportement mécanique des ouvrages à la mise en eau d'une part, et d'autre part suggéraient que cette prévision serait difficile à faire au moyen des méthodes de l'élasticité.

En effet, la fondation non linéaire et anisotrope, serait soumise à un système d'efforts, dus à la mise en eau, assez éloigné du système d'efforts dus à la construction. Un ensemble de caractéristiques élastiques apparentes, calé sur la réponse aux efforts de construction, ne semblait pas très adapté pour prévoir la réponse aux efforts de mise en eau.

Une étude fondée sur une modélisation mathématique incorporant la non-linéarité mécanique paraissait plus adaptée.

\section{3. ÉTUDE DU COMPORTEMENT DU BARRAGE PAR LE MODỀLE MATHÉMATIQUE}

\subsection{Présentation de la loi TRIADH}

\subsubsection{Généralités}

La loi de comportement TRIADH, mise au point à l'occasion de ces calculs, est une loi non linéaire adaptée aux matériaux de remblai (BERTRAND, LINO . 1985).

Tableau III

Contraintes évaluées dans le béton de la paroi à la cote 703 (en MPa)

\begin{tabular}{|c|c|c|c|c|}
\cline { 2 - 5 } \multicolumn{1}{c|}{} & \multicolumn{2}{c|}{ PAREMENT AMONT } & \multicolumn{2}{c|}{ PAREMENT AVAL } \\
\hline Direction & $\begin{array}{c}\text { Contrainte } \\
\text { totale }\end{array}$ & $\begin{array}{c}\text { Pression } \\
\text { interstitielle }\end{array}$ & $\begin{array}{c}\text { Contrainte } \\
\text { totale }\end{array}$ & $\begin{array}{c}\text { Pression } \\
\text { interstitielle }\end{array}$ \\
\hline Verticale & 1,82 & 0,28 & 2,46 & 0,17 \\
Amont-aval & 0,84 & 0,28 & 0,84 & 0,17 \\
R.D.-R.G. & 0,94 & 0,28 & 1,08 & 0,17 \\
\hline
\end{tabular}


Elle dérive de la loi A.D.H., mise au point dans les laboratoires de l'École Centrale de Paris, qui se situe elle-même dans la lignée des modèles de Cambridge (Granta Graval et Cam-Clay). C'est une loi incrémentale isotrope, élastoplastique, avec écrouissage. Elle rend compte du comportement des différents types de sols, qu'ils soient à dominante graveleuse ou argileuse. Elle est particulièrement adaptée à la représentation de chargements dont le caractère cyclique n'est pas déterminant. En particulier, elle permet de représenter le comportement des matériaux de remblai et de fondation lors des différentes phases de construction et de mise en eau d'un barrage.

La loi TRIADH suppose l'existence d'un seuil de plasticité défini dans l'espace des contraintes (fig. 18). A l'intérieur du seuil, le comportement du matériau est élastique. Sur le seuil, toute modification de l'état de contrainte n'amenant pas à un retour dans le domaine élastique provoque une part de déformations plastiques, la modification des variables d'écrouissage, et le déplacement du seuil avec l'état de contrainte.

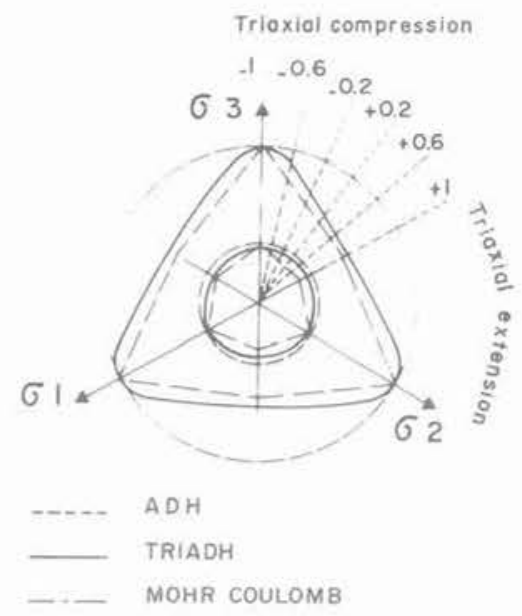

Fig. 18. - Surface de rupture dans le plan octaédral.

\subsubsection{Variables d'écrouissage du modèle}

Deux variables d'écrouissages sont prises en compte dans le modèle pour représenter l'histoire des sollicitations auquelles a été soumis le matériau. Elles sont liées aux déformations plastiques subies.

La première de ces variables représente l'écrouissage volumique. Elle est liée aux variations de densité sèche du matériau.

La seconde représente l'écrouissage déviatoire. Elle est liée aux déformations irréversibles de cisaillement.

\subsubsection{Invariants des contraintes}

Il est courant de formuler les modèles de comportement des matériaux isotropes en mécanique des sols en utilisant les trois invariants suivants ou des formes proportionnelles :

$$
\begin{aligned}
& \mathrm{p}=\frac{1}{3} \operatorname{Tr}(\sigma) \\
& \mathrm{q}=\left[\frac{3}{2} \operatorname{Tr}(\sigma-\mathrm{p} \delta)^{2}\right]^{1 / 2} \\
& \omega=\sin 3 \theta \text { avec } \theta=\frac{1}{3} \arcsin \left[\frac{27}{2} \frac{\operatorname{det}(\sigma-\mathrm{p} \delta)}{\mathrm{q}^{3}}\right]
\end{aligned}
$$

Leur signification est simple. Si l'on considère le domaine de l'espace des contraintes principales $\sigma_{1}>\sigma_{2}>\sigma_{3}$, les invariants $\mathrm{p}$, q et $\theta$ sont proportionnels aux coordonnées cylindriques $z, \rho$ et $\theta$ définis en prenant comme axe la trissectrice de l'espace, et comme plan $\theta=0$ le plan $\sigma_{2}=1 / 2\left(\sigma_{1}+\sigma_{3}\right)$.

L'invariant $\omega$ a été introduit dans le modèle TRIADH pour rendre compte de l'influence de la contrainte principale intermédiaire sur l'angle de frottement du matériau. Son importance apparait figure 19. Dans les modèles qui ont précédé $T R I A D H$, la non-prise en compte de cet invariant conduit à surestimer nettement langle de frottement résiduel du matériau sur des chemins différents de celui de l'essai triaxial en compression, notamment pour un matériau très frottant.

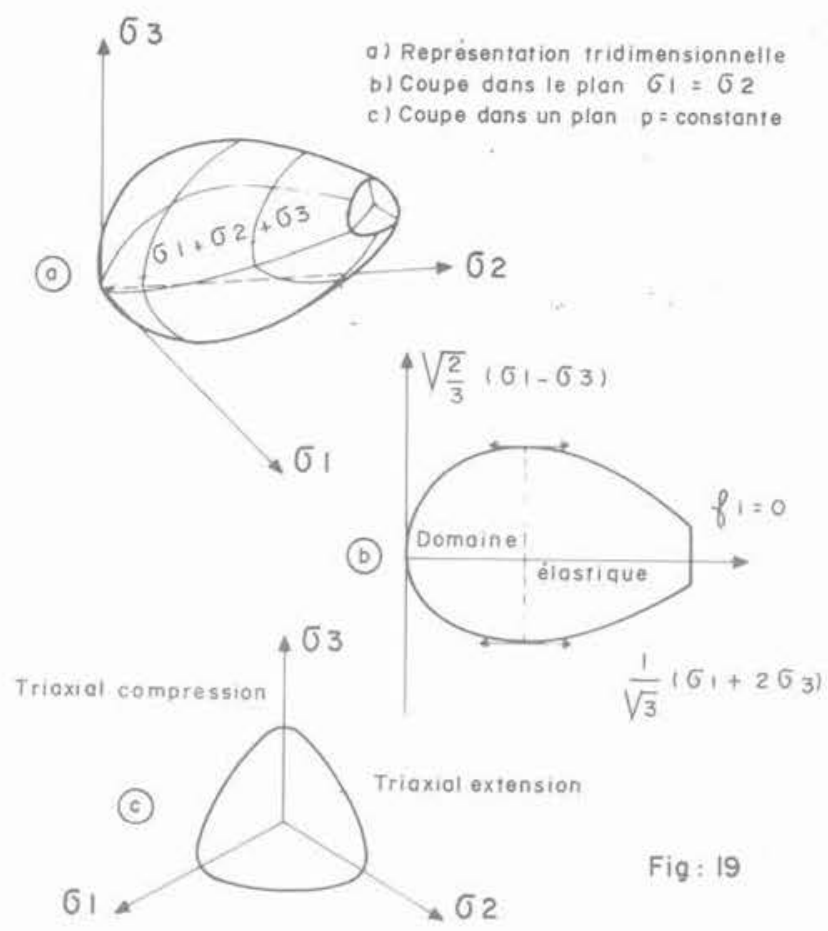

Fig. 19. - Forme du seuil de plasticité.

\subsubsection{Paramètres des modèles}

Sept paramètres sont à déterminer pour représenter un matériau par la loi TRIADH. Ce sont :

E, v paramètres du comportement élastique.

$\Phi_{\text {TC }}$ angle de frottement résiduel à l'essai triaxial en compression ;

B lié à la pente de l'essai œdométrique ; 
$\mathrm{P}_{\mathrm{CO}}$ lié à la densité initiale ;

a lié à la sensibilité à l'écrouissage déviatoire;

b lié au comportement au pic des contraintes;

$\alpha \quad$ lié à la limite du domaine élastique sur l'axe des pressions isotropes.

\subsubsection{Prédiction du modèle}

La figure 20 montre la comparaison entre les essais effectués par LADE et DUNCAN sur un sable en triaxial compression et triaxial extension, et la repré-

(a)

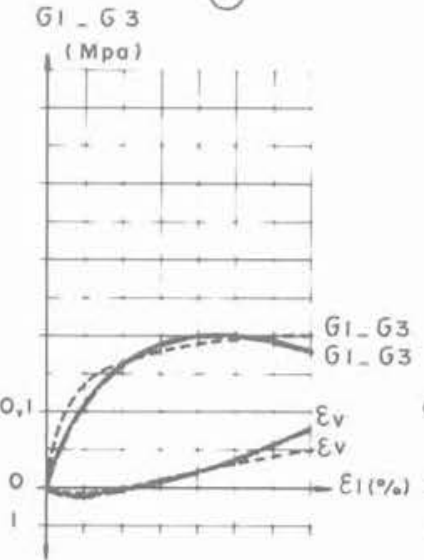

Simulation par le modele TRIADH

Simulation par le modèie $A D H$

-. Essais de LADE et DUNCAN (1973)

(b)

b) Triaxal extension.

Fig. 20. - Essais axisymétriques sur le sable de Monterey.

Etat noturel

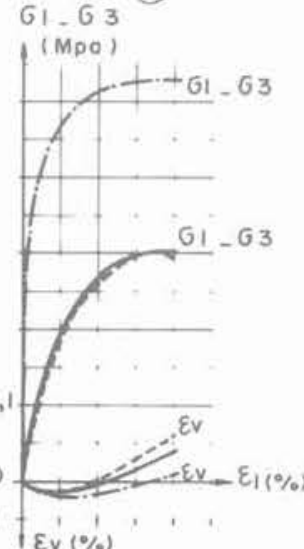




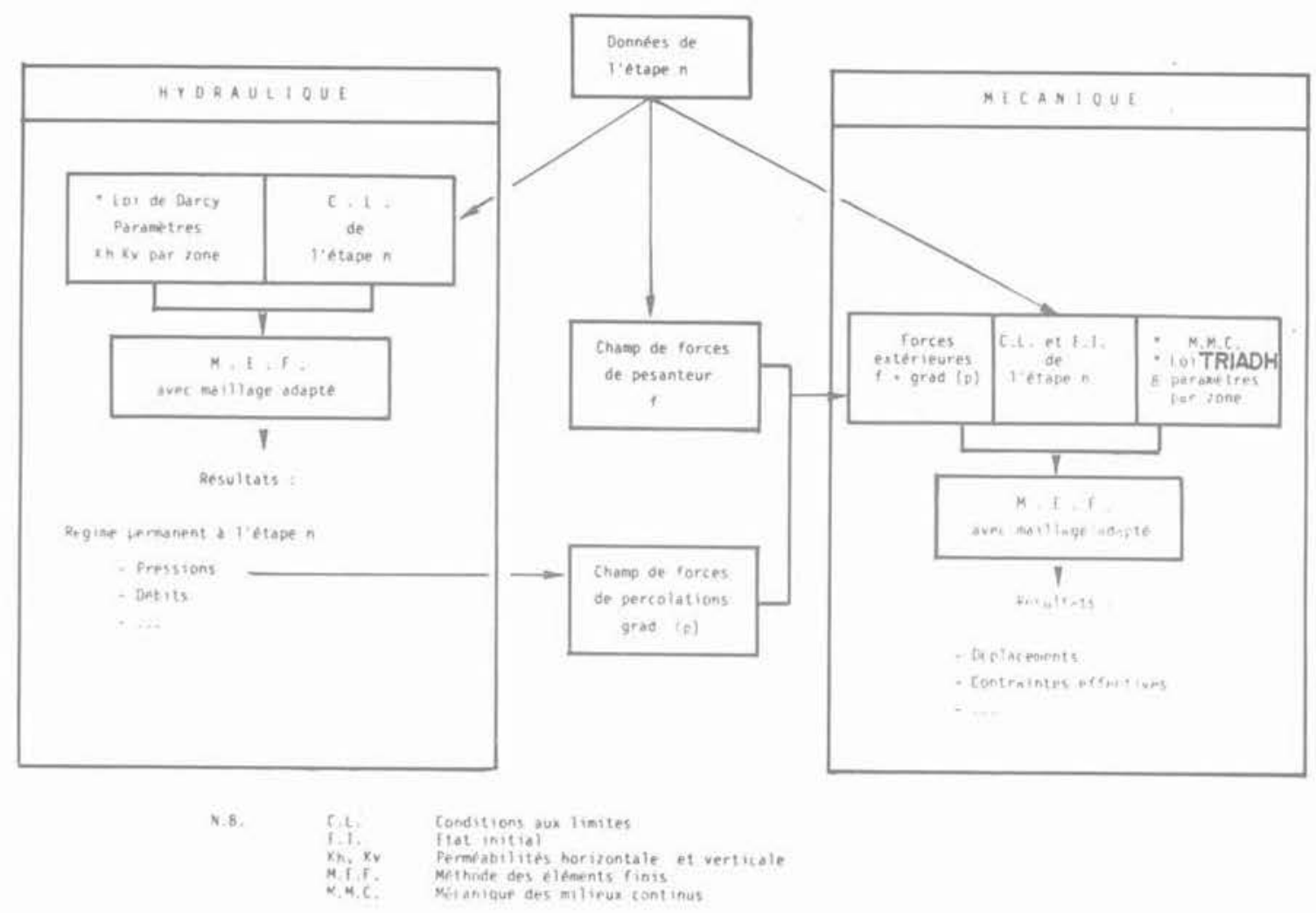

Fig. 22. - Schématisation d'une étape de calcul.

\subsubsection{Choix d'une modélisation}

Le modèle finalement choisi est donc semi-couplé (fig. 22) :

- les différents écoulements permanents régnant en fondation sont calculés indépendamment du comportement mécanique des matériaux;

- ils permettent de déduire les champs de forces de percolation dans chaque régime d'écoulement. Ces champs de forces sont introduits comme forces extérieures dans le calcul mécanique. Celui-ci s'effectue donc en contraintes effectives.

Le modèle géométrique construit pour résoudre les calculs par la méthode des éléments finis s'étend sur une grande longueur (fig. 8), de manière à éviter que le choix toujours délicat des conditions aux limites hydrauliques ou mécaniques n'influe trop sur les résultats. Il représente les différents éléments de l'ouvrage, en particulier la paroi moulée, la piste de chantier provisoire utilisée lors de la construction et les sept couches de matériaux distinguées en fondation.

\subsubsection{Distinction des matériaux}

\section{et calage des paramètres mécaniques}

Les sept couches distinguées en fondation sont le fruit de l'interprétation des mesures fournies par l'auscultation. Une première approche avait permis d'attribuer à chacune des couches des paramètres de comportement élastique (cf. § 2.2.2).
A partir de ces paramètres élastiques a été estimé un premier jeu de paramètres TRIADH, qui a ensuite été affiné de manière à permettre la meilleure représentation par le calcul des observations faites pendant les phases de construction.

Les figures 23 à 25 montrent que le modèle représente assez bien les phases de construction du barrage sur lequel il a été calé. Sur les figures 23 et 24 , on retrouve les différences de comportement entre les couches; notons que celles-ci ont été modélisées parallèles et d'épaisseur constante, alors que les mesures laissent voir qu'elles présentent probablement des variations modérées d'épaisseur selon les zones. La figure 25 indique la qualité du calage sur l'ensemble de l'emprise du barrage.

La valeur de ce calage autorisait à utiliser le modèle pour interpréter les observations faites pendant la construction, et prévoir ce qui adviendrait à la mise en eau.

\subsection{Résultats du calcul et interprétation}

\subsubsection{Phase de construction du barrage}

En fin de construction du barrage, après enlèvement de la piste de chantier, on a pu faire différentes constatations sur le massif de tête et le casque de la paroi moulée. On a noté d'une part des ouvertures de décol- 


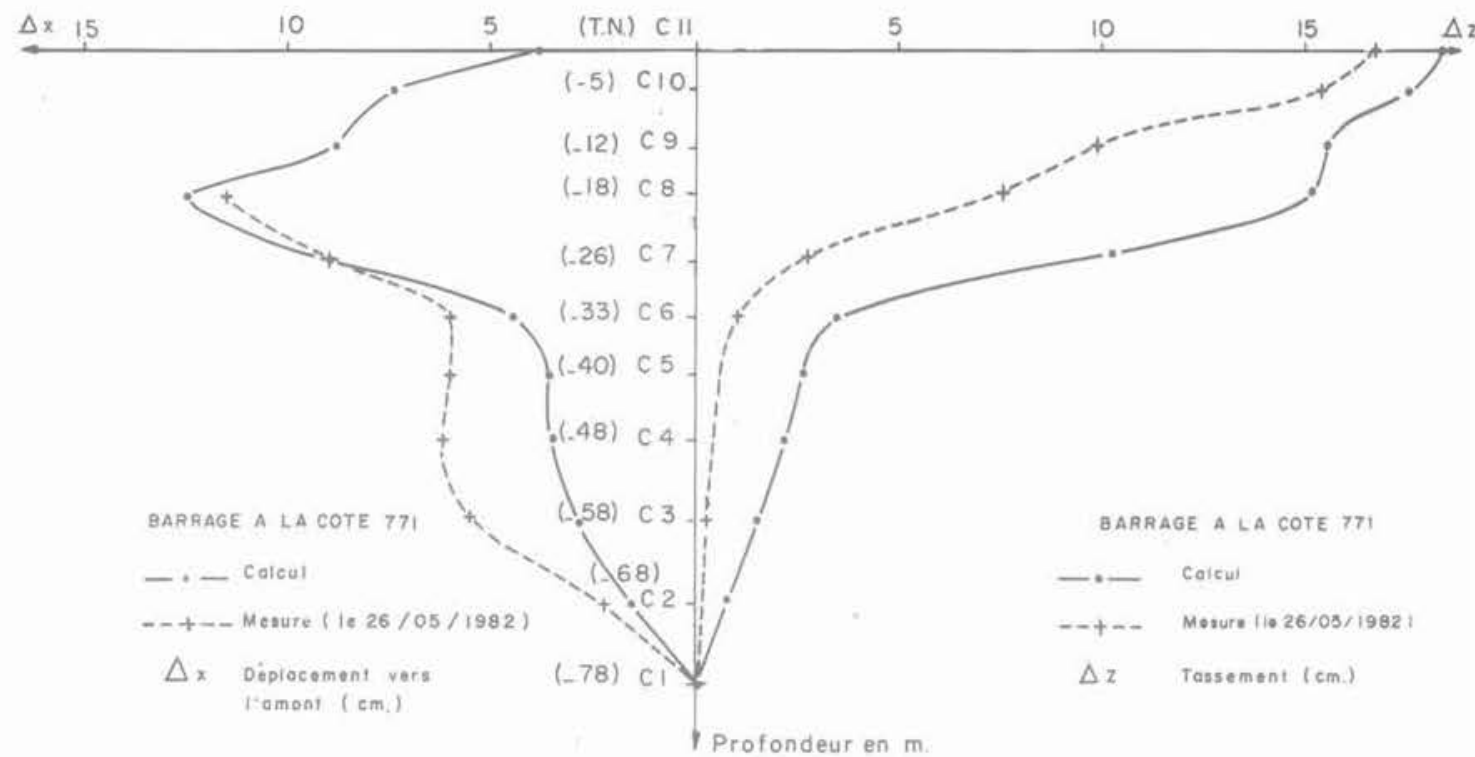

a

Fig. 23a. - Déplacements horizontaux calculés et mesurés au clinofor après la fin de la construction du barrage.

Fig. 23b. - Tassements calculés et mesurés au distofor amont après la fin de la construction du barrage.
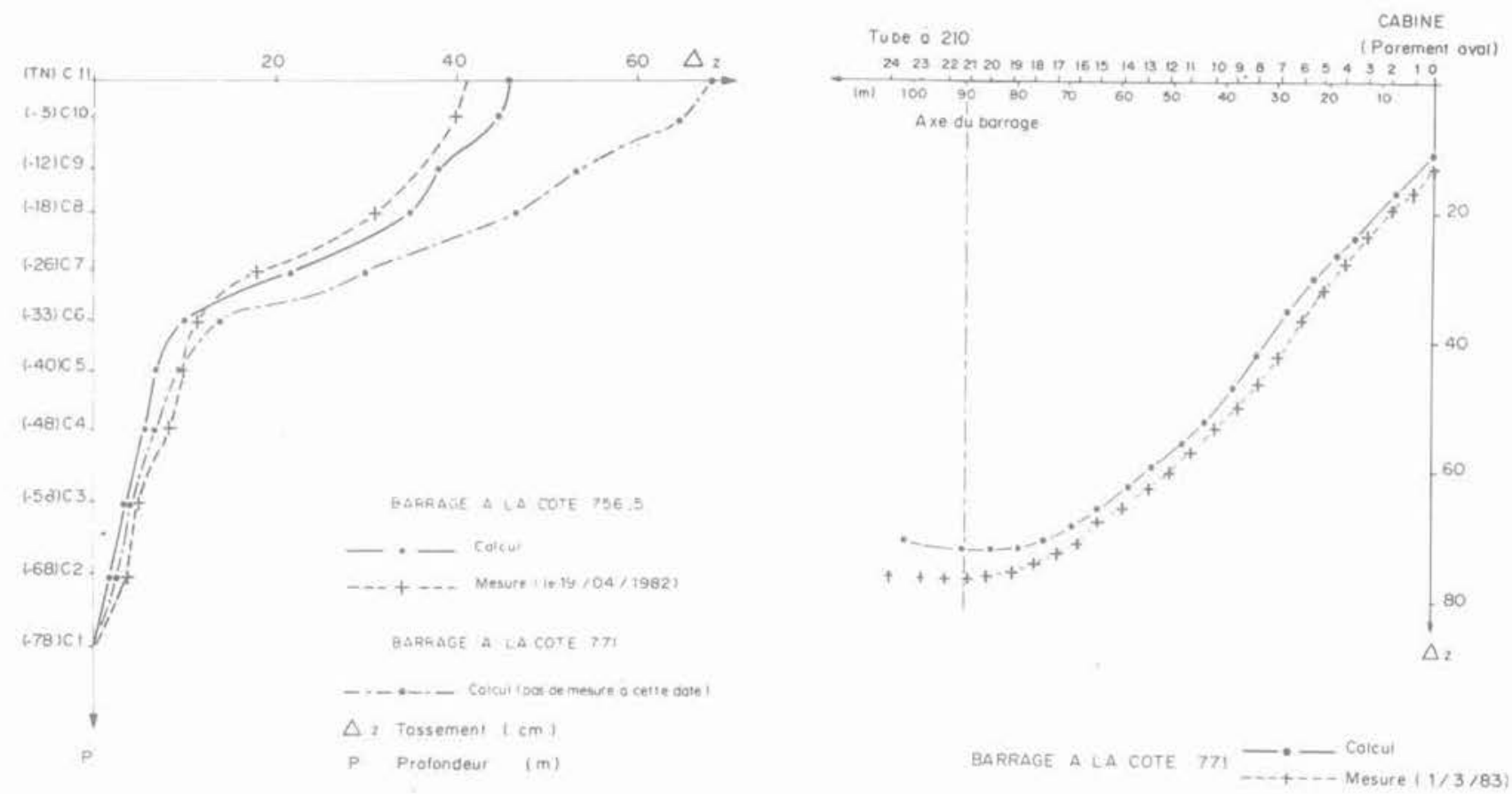

$\Delta z$ Tossement $(\mathrm{cm})$

Fig : 25

Fig. 24. - Tassements calculés et mesurés au distofor aval après la fin de la construction du barrage.
Fig. 25. - Tassements calculés et mesurés au tube horizontal après prise en compte des tassements différés. 


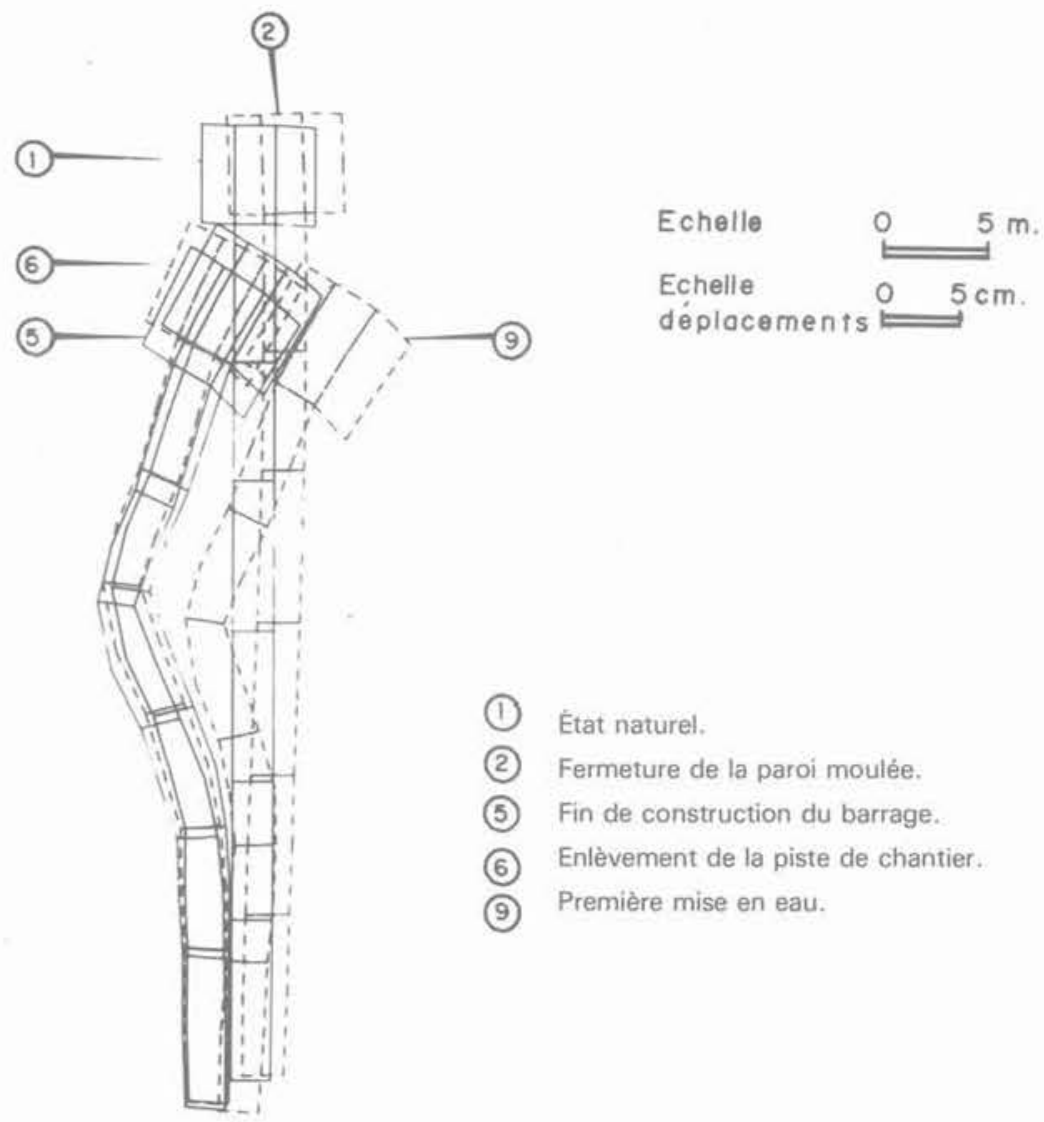

Fig. 26. - Barrage du Verney

Calcul TRIADH. Déformées de la paroi moulée.

lement entre le massif de tête et la fondation, d'autre part de petites fissures dans des plans amont-aval sur le casque de la paroi moulée. Le calcul a aidé à expliquer ces observations, et à mesurer leur importance.

Le calcul a permis de montrer que la contrainte verticale de contact entre fondation et massif de tête de la paroi moulée allait jusqu'à s'annuler lors de l'enlèvement de la piste de chantier, ce qui traduit l'ouverture de fissures de décollement. Le comportement élastique de la paroi moulée (dans cette gamme de déformations) contrastant avec la grande irréversibilité de comportement au déchargement de la fondation expliquent ce phénomène.

D'autre part, le calcul montre que la construction du barrage provoque un net basculement du massif de tête de la paroi (fig. 26). Ce basculement est gêné en rive par les injections effectuées dans les alluvions. Entre le basculement au centre de la vallée et le blocage en rive, le casque de béton armé de la paroi moulée a été soumis à un effort de torsion, source des microfissures constatées.

\subsubsection{Mise en eau}

\section{Prédiction du calcul}

D'après le calcul, les sollicitations imposées aux différents éléments du barrage devaient rester largement admissible à la mise en eau.
La figure 26 montre que les déformations de la paroi moulée sont en grande partie prises pendant la construction, et n'augmentent pas considérablement à la mise en eau. Le point de la paroi le plus sollicité vis-àvis du critère d'élasticité de béton plastique présenté au $\S 2.3 .1$. reste très en deçà de la limite ainsi que le montre la figure 27.

De faibles tractions se produisent dans la partie amont du massif de tête (fig. 28). L'étroitesse de la zone tendue, la faible valeur de traction et la présence du casque en béton armé éliminent les risques de fracturation hydraulique.

Le masque en béton bitumineux est partout comprimé, sauf en partie supérieure où il est légèrement tendu (fig. 29). Les valeurs des déformations mesurées sont très inférieures aux valeurs admissibles.

\section{Comparaison avec les mesures}

Le système d'auscultation a été complété à la mise en eau par (fig. 30) :

- des relevés topographiques en différents points du parement aval;

- un tassomètre, un élongamètre et des clinomètres installés sur le casque de la paroi moulée. 


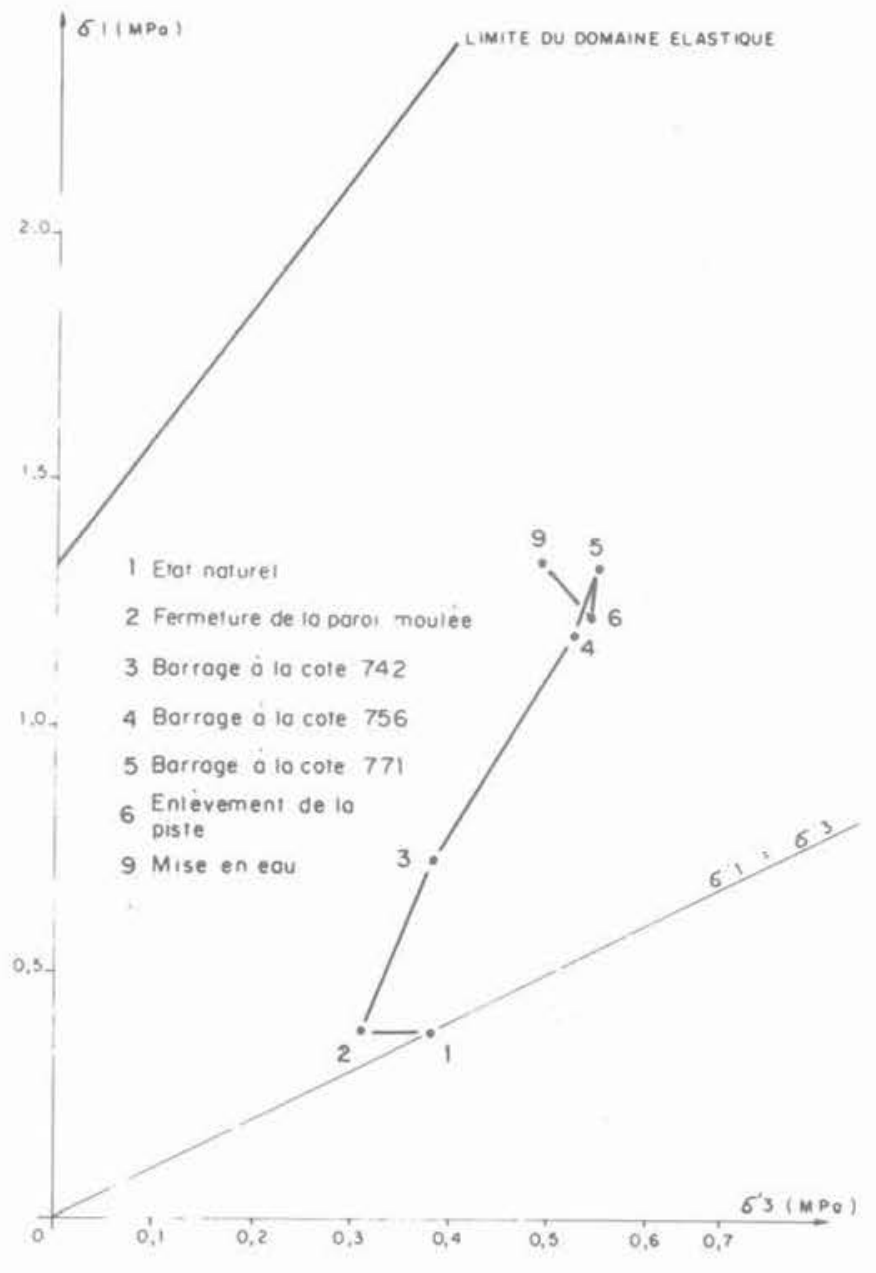

Fig. 27. - Evvolution des contraintes extrémales du point de la paroi moulée le plus proche de la rupture en compression.

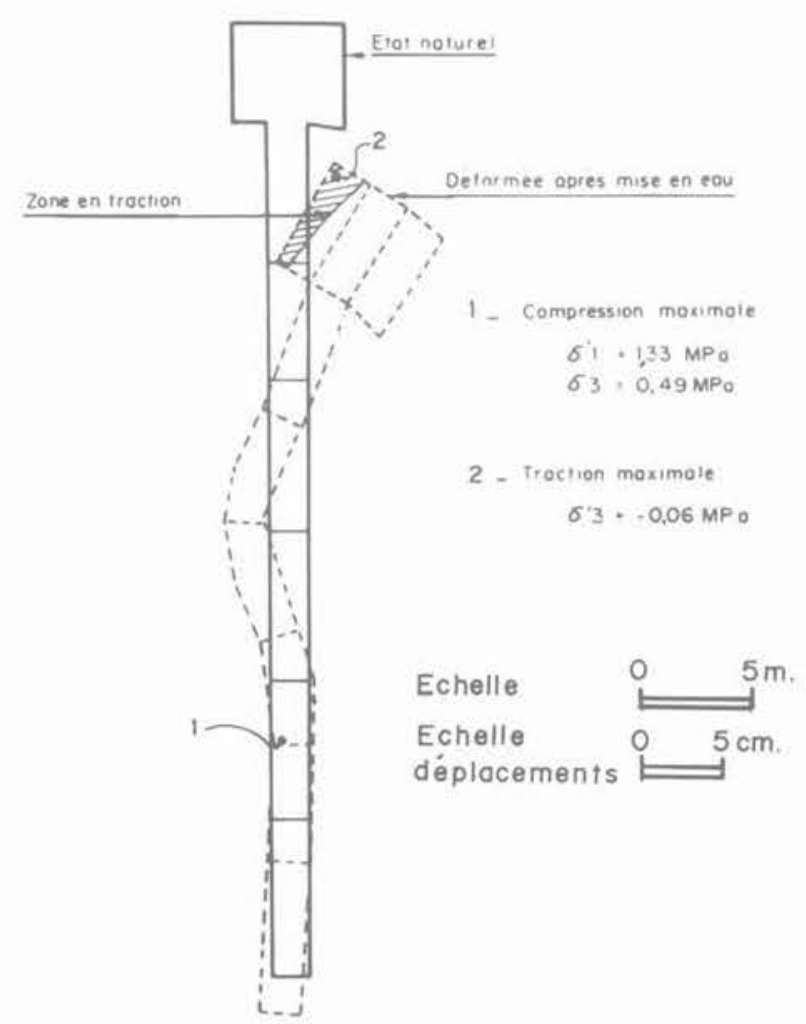

Fig. 28. - Tractions et compressions maximales dans la paroi moulée après la mise en eau.
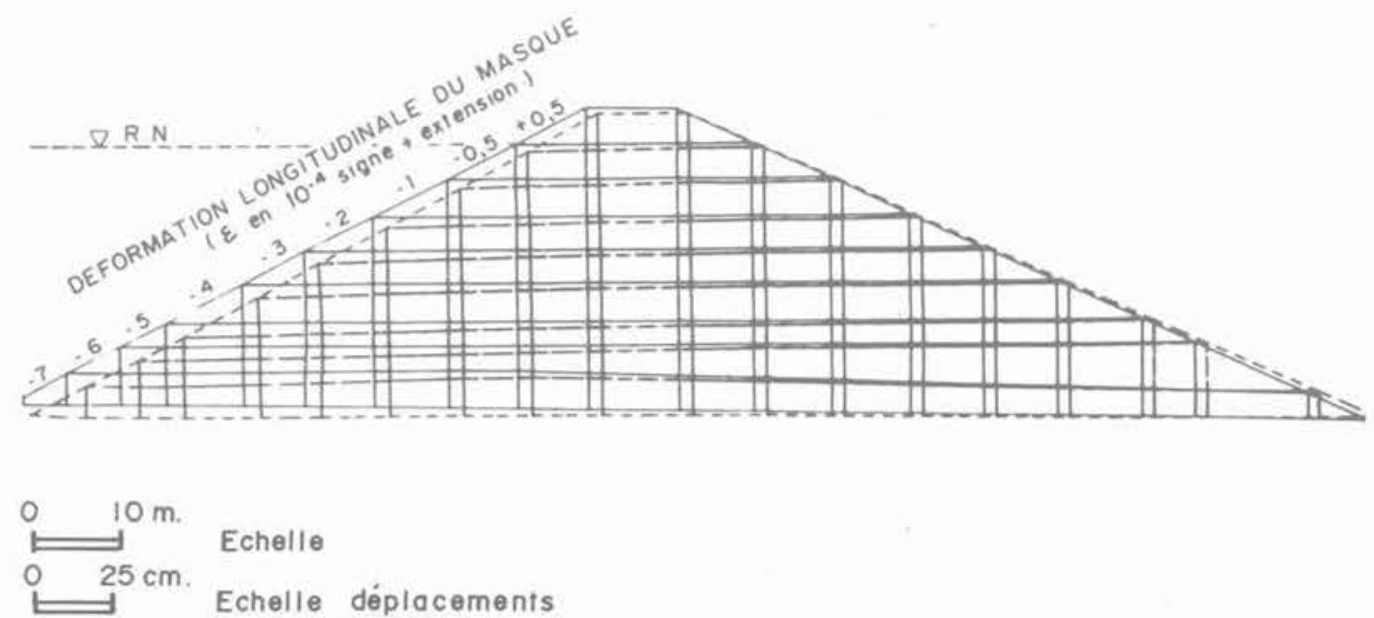

Fig. 29. - Barrage du Verney - Calcul TRIADH

Déformation du barrage à la mise en eau. 


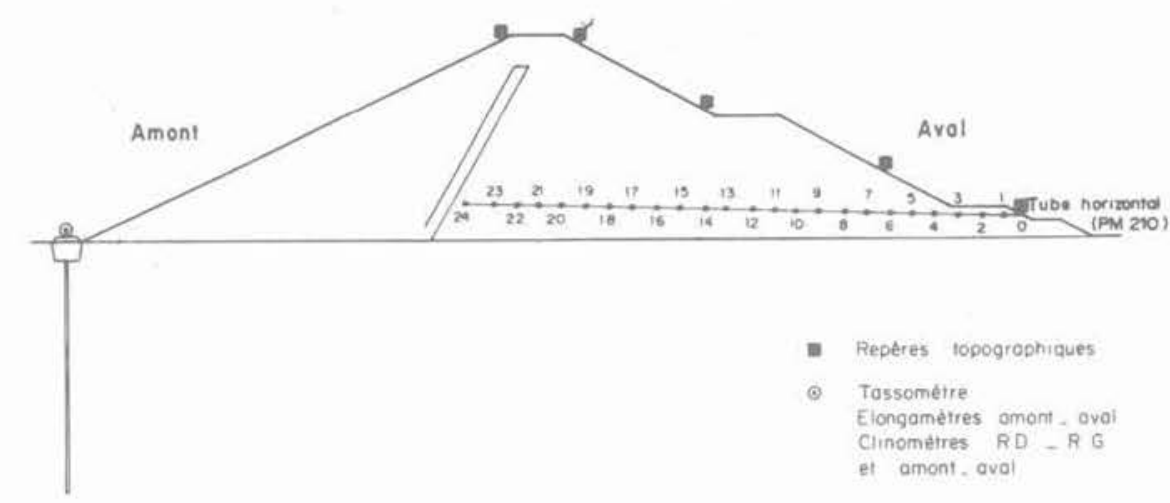

Fig. 30. - Auscultation des déplacements durant la mise en eau.

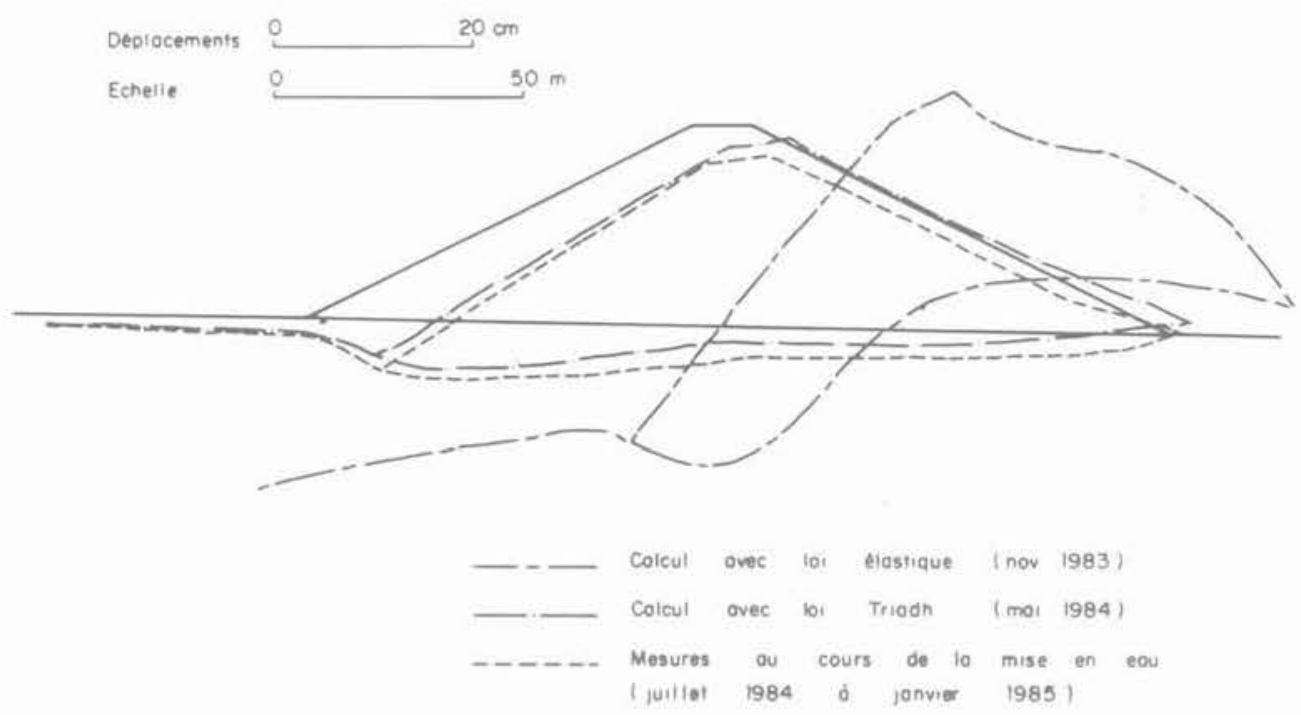

Fig. 31. - Barrage du Verney - Mise en eau

Déformées du barrage suivant les calculs et suivant les mesures.

Au cours de la mise en eau, les prévisions du calcul ont servi de repère pour contrôler la normalité des résultats de mesure. Ainsi, on n'a pas été surpris de constater que les points situés dans l'axe du barrage commençaient par tasser, avant de se réhausser puis, tasser à nouveau. Le calcul avait montré ce phénomène surprenant, et permis de l'expliquer par les effets opposés de la pression de l'eau sur le masque et des gradients verticaux dans la fondation du barrage.

En fin de mise en eau, on a pu comparer prévision et calcul (fig. 31) et constater leur remarquable concordance.

Cette qualité du calcul est soulignée par la comparaison que l'on peut en faire avec les prévisions d'un calcul effectué peu avant le calcul TRIADH, dans lequel les matériaux étaient représentés au moyen des lois de l'élasticité linéaire. Ce précédent calcul était calé sur les mêmes phases de construction que le calcul TRIADH, et le calage était d'aussi bonne qualité.

La figure 31 illustre l'inadaptation des lois de l'élasticité pour représenter le comportement des matériaux de fondation, même pour des chemins de contraintes ne faisant pas apparaître de traction. Elle montre au contraire la qualité de prévision que permettent les lois non linéaires du type de la loi TRIADH.

\section{CONCLUSION}

L'ensemble des travaux d'interprétation et de calcul relaté dans cet exposé s'est déroulé pendant plus de quatre ans.

A chaque nouvelle étape de construction de l'aménagement, les nouvelles mesures collectées ont permis 
d'affiner l'image que l'on se faisait de la fondation. La richesse et la qualité du dispositif d'auscultation hydraulique et mécanique ont permis d'aboutir à une représentation suffisamment précise de la fondation, malgré sa complexité.

La qualité de cette représentation de la fondation jointe à celle de la loi de comportement TRIADH, a permis la construction d'un modèle de calcul. Ce modèle a été d'une grande aide pour le projeteur, et s'est avéré un véritable outil de prédiction et non un simple exercice académique.

\section{BIBLIOGRAPHIE}

BERTRAND J., LINO M. (1985), TRIADH and its application : calculating to predict the behaviour of Verney Dam during first impounding, Proc. 11th I.C.S.M.F.E., San Francisco, August 12-16, 1985.
COSTE J., POST G., TARDIEU B. (1982), Barrage du Verney - Projet et début de réalisation, Travaux, $\mathrm{n}^{\circ} 564$, mars $1982,46-50$.

LINO M. (1980), Un modèle de matériau microfissuré, Revue Française de Géotechnique, $n^{\circ} 11$, mai 1980 , 28-41.

POULOS H.G., DAVIS E.H. (1974), Elastic solutions for soil and rock mechanics, Wiley \& Sons, New York.

TARDIEU B. (1984), Barrage du Verney - Conception et réalisation de l'écran d'étanchéité : auscultation et calculs, Comité Français des Grands Barrages, Paris, mai 1984

TARDIEU B. (1981), Rhéologie du béton plastique Exemple du barrage du Verney, Colloque Technique de la C.F.G.B., 16 janvier 1981.

Groupe de travail du C.F.G.B., Auscultation des barrages en remblai. Enseignements déduits des comparaisons entre mesures et calculs par la méthode des élé. ments finis, XVe C.I.G.B., Lausanne, 1985. 
